\title{
Asymmetric Allylboration of Ketones Catalyzed by Chiral Diols
}

\author{
Sha Lou, Phil N. Moquist, and Scott E. Schaus* \\ Department of Chemistry, Center for Chemical Methodology and Library Development at \\ Boston University (CMLD-BU), Life Science and Engineering Building, Boston University \\ 24 Cummington Street, Boston, Massachusetts, 02215
}

\section{Supporting Information}

\section{Table of Contents}

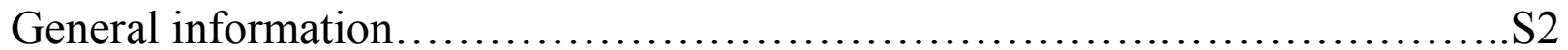

Preparation of allyldiisopropoxyborane..................................S2

Preparation of crotyldiisopropoxyborane.................................S3

General procedure for asymmetric allylboration of ketones...................S4

Analytical data for allylboration products 11a-o, 13a-b......................S5

Positive non-linear effect................................................... 11

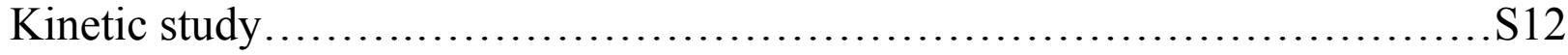

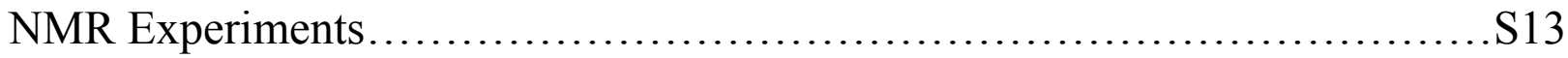

HPLC traces for 11a-o, 13a-b........................................... 18 
General Information. All ${ }^{1} \mathrm{H}$ NMR, and ${ }^{13} \mathrm{C}$ NMR spectra were recorded using Varian Unity Plus $400\left(93.94 \mathrm{kG},{ }^{1} \mathrm{H} 400 \mathrm{MHz}\right)$ or Varian Gemini $300\left(70.5 \mathrm{kG},{ }^{13} \mathrm{C} 75 \mathrm{MHz}\right)$ spectrometers at ambient temperature in $\mathrm{CDCl}_{3}$. Chemical shifts are reported in parts per million as follows: chemical shift, multiplicity $(\mathrm{s}=$ singlet, $\mathrm{d}=$ doublet, $\mathrm{t}=$ triplet, $\mathrm{q}=$ quartet, $\mathrm{m}=$ multiplet, $\mathrm{br}=$ broad), coupling constant, and integration. Infrared spectra were recorded on a Nicolet Nexus 670 FT-IR ESP spectrophotometer. Optical rotations were recorded on an AUTOPOL III digital polarimeter at $589 \mathrm{~nm}$, and were reported as $[\alpha]_{\mathrm{D}}$ (concentration in grams/100 $\mathrm{mL}$ solvent). Analytical thin layer chromatography was performed using EMD $0.25 \mathrm{~mm}$ silica gel $60-\mathrm{F}$ plates. Flash column chromatography was performed on Sorbent Technologies $60 \AA$ silica gel. Chiral HPLC analysis was performed using an Agilent 1100 series HPLC or Waters Breeze HPLC System with a diode array detector. Chiral columns include Chiralcel ${ }^{\circledR}$ OD (Chiral Technologies Inc., $24 \mathrm{~cm} \times 4.6 \mathrm{~mm}$ I.D.), Chiralcel ${ }^{\circledR} \mathrm{OD}-\mathrm{H}$ (Chiral Technologies Inc., $15 \mathrm{~cm} \times 4.6 \mathrm{~mm}$ I.D.), Chiralpak ${ }^{\circledR}$ AD-H (Chiral Technologies Inc., $15 \mathrm{~cm} \times 4.6 \mathrm{~mm}$ I.D.), and Chiralpak ${ }^{\circledR}$ AD-H (Chiral Technologies Inc., $25 \mathrm{~cm} \times 4.6 \mathrm{~mm}$ I.D.). Chiral GC analysis was performed using an Agilent 6850 series GC system and Chiraldex B-DM as chiral column (Chiral Technologies Inc., $30 \mathrm{~m} \times$ $0.25 \mathrm{~mm}$ I.D.). All reactions were performed under argon, in oven dried glassware with magnetic stirring. Chiral diols, including (-)-TADDOL 1, (+)-hydrobenzoin 2, (+)-diethyltartrate 3, $(S)$ BIPHEN 4, $(S)$-BINOL 5a and 6,6'-Br-BINOL 7, were purchased from Sigma-Aldrich, STREM, and Alpha Aesar and used without further purification. Diols 5b, 5c, 6a, 6b were prepared according to literature procedure. ${ }^{1}$ Monomethyl ether $\mathbf{8}$ was prepared by alkylating one alcohol using $\mathrm{NaH}$ and $\mathrm{CH}_{3} \mathrm{I}$ as previously described in the literature. ${ }^{1 \mathrm{c}}$ Kinetic parameters for the asymmetric allylboration reaction were determined by in situ monitoring of the disappearing of acetophenone (9a) at $1690.27 \mathrm{~cm}^{-1}$ using a ReactIR 4000 system (Mettler Toledo-AutoChem). The ReactIR 4000 system, running software version 3.1, was fitted with a FiberConduit and a 6 mm DiComp Probe. IR spectra, comprised of 64 scans per spectrum, were collected every one minute at a resolution of $8 \mathrm{~cm}^{-1}$.

\section{Preparation of allyldiisopropoxyborane 10a}

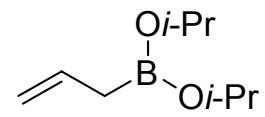

A $250 \mathrm{~mL}$ pressure-equilized addition funnels charged separately, one with a $1.0 \mathrm{M}$ solution of triisopropyl borate $(154.2 \mathrm{~mL}, 154.2 \mathrm{mmol})$ in diethyl ether and the other with a $1.0 \mathrm{M}$ solution of allylmagnesium bromide $(150.4 \mathrm{~mL}, 150.4 \mathrm{mmol})$ in diethyl ether, were attached to a dry $1 \mathrm{~L}$ three neck flask under Ar. The flask was cooled to $-78{ }^{\circ} \mathrm{C}$ and both reagents were added dropwise simultaneously over 1 hour with vigorous stirring. After addition was completed, the reaction mixture was slowly warmed up to $0^{\circ} \mathrm{C}$ and stirred for 3 hour. The mixture was cooled to $-78{ }^{\circ} \mathrm{C}$ and $2.0 \mathrm{~N} \mathrm{HCl}(150.4 \mathrm{~mL}, 150.4 \mathrm{mmol})$ in ether solution was slowly added over 30 minutes. The solution was warmed to $0{ }^{\circ} \mathrm{C}$ and stirred for 1 hour. Precipitate was removed by filtration and the filtrate was concentrated to $100 \mathrm{~mL}$. The bottom oily layer was removed and the remaining solvent was concentrated under reduced pressure. The impure oil was pipetted from the residue and run through a small plug of $\mathrm{Na}_{2} \mathrm{SO}_{4}$. Oil was cooled to $-78{ }^{\circ} \mathrm{C}$ and filtered

(1) (a) Wipf, P.; Jung, J-K. J. Org. Chem. 2000, 20, 6319. (b) McDougal, N. T.; Trevellini, W. L.; Rodgen, S. A.; Kliman, L. T.; Schaus, S. E. Adv. Synth. Catal. 2004, 346, 1231. (c) Liu, H.; Liu, Y.; Zhu, C.; Liu, M.; Wang, C.; Chen, C.; Xi, F. Synthetic Metals 2002, 131, 135. 
through a small plug of $\mathrm{Na}_{2} \mathrm{SO}_{4}$. The colorless oil was then dissolved in toluene to make $1.0 \mathrm{M}$ solution. The spectral data was in agreement with reported values. ${ }^{2}$

\section{Preparation of Crotyldiisopropoxyborane ${ }^{3}$ (E)-But-2-enyldiisopropoxyborane 12a}<smiles>C/C=C/CP(CCC)O[Bi]</smiles>

A $250 \mathrm{~mL}$ three-neck round bottom flask was charged with a magnetic stir bar and a thermometer. $100 \mathrm{~mL} \mathrm{THF}$ and $\mathrm{KO} t$ - $\mathrm{Bu}(4.65 \mathrm{~g}, 41.5 \mathrm{mmol})$ were added. The mixture was flushed with Ar and cooled to $-78^{\circ} \mathrm{C}$. Trans-2-butene (2.5 g, $\left.44 \mathrm{mmol}\right)$, condensed into a rubberstoppered test tube kept at $-78^{\circ} \mathrm{C}$, was added via cannula. $n$-BuLi (1.6 M in hexane, $26 \mathrm{~mL}, 41.5$ $\mathrm{mmol}$ ) was added dropwise over 1 hour with an addition funnel so that the internal temperature did not rise at all. After completion of the addition, the reaction mixture was allowed to warm until the internal temperature reached $-52{ }^{\circ} \mathrm{C}$. The solution was maintained at $-52{ }^{\circ} \mathrm{C}$ for 15 minutes, and then cooled back to $-78{ }^{\circ} \mathrm{C}$. Triisopropylborate $(10.5 \mathrm{~mL}, 45.6 \mathrm{mmol})$ was then added dropwise over 30 minutes through an addition funnel. The reaction mixture was maintained at $-78{ }^{\circ} \mathrm{C}$ for 2 hours and then rapidly poured into a $500 \mathrm{~mL}$ separation funnel containing $80 \mathrm{~mL}$ of $1 \mathrm{~N} \mathrm{HCl}$ saturated with $\mathrm{NaCl}$. The aqueous layer is adjusted to $\mathrm{pH} 1$ by using $1 \mathrm{~N} \mathrm{HCl}$. The organic phases were separated, and the aqueous layer was extracted with additional $\mathrm{Et}_{2} \mathrm{O}(3 \times 50 \mathrm{~mL})$. The combined extracts were dried with $\mathrm{Na}_{2} \mathrm{SO}_{4}$ and filtered. The filtrate was concentrated to about $100 \mathrm{~mL}$ and was treated with isopropyl alcohol $(6.34 \mathrm{~mL}, 83$ $\mathrm{mmol})$ and $3 \AA$ molecular sieves $(8.0 \mathrm{~g})$. The mixture was stirred under Ar for 24 hours at room temperature. Stirring was discontinued and the clear supernatant ether layer was transferred in to another flask, the molecular sieves were washed once again with ether $(100 \mathrm{~mL})$. The ethereal extracts were combined and ether was removed under reduced pressure. The flask having clear liquid left was put on high vacuum pump to pump off any volatile solvent. Some white solid was formed during the course and the liquid was quickly filtered though a cotton plug and stored as 1.0 M toluene solution at $0{ }^{\circ} \mathrm{C}$. This solution was used in the crotylboration reaction without any further purification. The analytical sample was obtained by distillation under reduce pressure (90 ${ }^{\circ} \mathrm{C}, 0.1$ Torr) and the pure sample was sensitive to moisture. ${ }^{1} \mathbf{H}$ NMR (400 $\mathrm{MHz}, \mathrm{CDCl}_{3}$ ): $\delta 5.43(\mathrm{~m}, 1 \mathrm{H}), 5.30(\mathrm{~m}, 1 \mathrm{H}), 4.42-4.27(\mathrm{~m}, 2 \mathrm{H}), 1.61(\mathrm{br} \mathrm{d}, J=6.0 \mathrm{~Hz}, 2 \mathrm{H}), 1.56(\mathrm{br} \mathrm{d}, J=6.0$ $\mathrm{Hz}, 3 \mathrm{H}), 1.10(\mathrm{~d}, J=6.0 \mathrm{~Hz}, 12 \mathrm{H}) ;{ }^{13} \mathrm{C}$ NMR (75.0 MHz, $\left.\mathrm{CDCl}_{3}\right): \delta 129.0,128.2,69.1,24.6$, $24.5,21.5,18.1$.

\section{(Z)-But-2-enyldiisopropoxyborane $12 b$}<smiles>C/C=C\CB(O[Tl])OCC</smiles>

A $250 \mathrm{~mL}$ three-neck round bottom flask was charged with a magnetic stir bar and a thermometer. $100 \mathrm{~mL}$ THF and $\mathrm{KO} t-\mathrm{Bu}(4.65 \mathrm{~g}, 41.5 \mathrm{mmol})$ were added in. The mixture was flushed with $\mathrm{Ar}$ and cooled to $-78^{\circ} \mathrm{C}$. Cis-2-butene $(2.50 \mathrm{~g}, 44 \mathrm{mmol})$, condensed into a rubber-

(2) Brown, H. C.; Rachela, U. S.; Pellechia, P. J. J. Org. Chem. 1990, 55, 1868.

(3) Roush, W. R.; Ando, K.; Powers, D. B.; Palkowitz, A. D.; Halterman, R. L. J. Am. Chem. Soc. 1990, 112, 6339. 
stoppered test tube kept at $-78^{\circ} \mathrm{C}$, was added via cannula. $n$-BuLi (1.6 M in hexane, $26 \mathrm{~mL}, 41.5$ mmol) was added dropwise over 0.5 hour with an addition funnel. After completion of the addition, the reaction mixture was allowed to warm until the internal temperature reached $-25^{\circ} \mathrm{C}$. The solution was maintained at $-25^{\circ} \mathrm{C}$ for 30 minutes, and then cooled back to $-78{ }^{\circ} \mathrm{C}$. Triisopropylborate $(10.5 \mathrm{~mL}, 45.6 \mathrm{mmol})$ was then added dropwise over 30 minutes through an addition funnel. The reaction mixture was maintained at $-78{ }^{\circ} \mathrm{C}$ for 2 hours and then rapidly poured into a $500 \mathrm{~mL}$ separation funnel containing $80 \mathrm{~mL}$ of $1 \mathrm{~N} \mathrm{HCl}$ saturated with $\mathrm{NaCl}$. The aqueous layer is adjusted to $\mathrm{pH} 1$ by using $1 \mathrm{~N} \mathrm{HCl}$. The organic phases are separated, and the aqueous layer is extracted with additional $\mathrm{Et}_{2} \mathrm{O}(3 \times 50 \mathrm{~mL})$. The combined extracts are dried with $\mathrm{Na}_{2} \mathrm{SO}_{4}$ and filtered. The filtrated was concentrated to about $100 \mathrm{~mL}$ and was treated with isopropyl alcohol $(6.34 \mathrm{~mL}, 83 \mathrm{mmol})$ and $3 \AA$ molecular sieves $(8.0 \mathrm{~g})$. The mixture was stirred under Ar for 24 hours at room temperature. Stirring was discontinued and the clear supernatant ether layer was transferred in to another flask, the molecular sieves were washed once again with ether $(100 \mathrm{~mL})$, and the ethereal extracts were combined and ether was removed under reduced pressure. The flask having clear liquid left was put on high vacuum pump to pump off any volatile solvent. Some white solid was formed during the course and the liquid was quickly filtered though a cotton plug and stored as $1.0 \mathrm{M}$ Toluene solution at $0{ }^{\circ} \mathrm{C}$. This solution was used in the crotylboration reaction without any further purification. The analytical sample was obtained by distillation under reduce pressure $\left(90^{\circ} \mathrm{C}, 0.1\right.$ Torr $)$ and the pure sample was sensitive to moisture. ${ }^{1} \mathbf{H}$ NMR (400 MHz, $\left.\mathrm{CDCl}_{3}\right): \delta 5.52(\mathrm{~m}, 2 \mathrm{H}), 4.47(\mathrm{~m}, 1 \mathrm{H}), 4.36(\mathrm{~m}, 1 \mathrm{H}), 1.64$ (br d, $J=7.2 \mathrm{~Hz}, 2 \mathrm{H}), 1.58$ (br d, $J=6.0 \mathrm{~Hz}, 3 \mathrm{H}), 1.18$ (d, $J=6.0 \mathrm{~Hz}, 12 \mathrm{H}) ;{ }^{13} \mathbf{C}$ NMR $(75.0 \mathrm{MHz}$, $\left.\mathrm{CDCl}_{3}\right): \delta 126.9,124.8,68.1,24.8,24.7,20.3,12.7$.

\section{General Procedure for Asymmetric Allylboration of Ketones.}
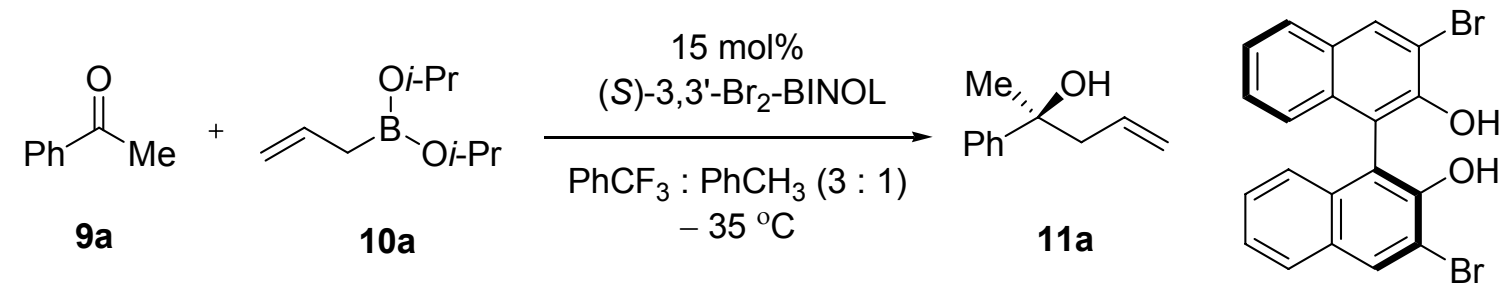

A $10 \mathrm{~mL}$ one neck round bottom flask was charged with stir bar and flushed with Ar. To this flask was added 3,3'-dibromo-1,1'-bi-2-naphthol (33 mg, $0.075 \mathrm{mmol}), \alpha, \alpha, \alpha$-triflurotoluene $(3.75 \mathrm{~mL})$, toluene $(0.75 \mathrm{~mL})$ and acetophenone $(87.5 \mu \mathrm{L}, 0.75 \mathrm{mmol})$. The mixture was stirred under Ar at room temperature for 5 minutes and cooled at $-35^{\circ} \mathrm{C}$. The allyldiisopropoxyborane toluene solution $(500 \mu \mathrm{L}, 0.5 \mathrm{mmol}, 1.0 \mathrm{M}$ solution) was syringed in dropwise. The reaction mixture was stirred at $-35^{\circ} \mathrm{C}$ for $15 \mathrm{~h}$. and quenched with saturated $\mathrm{NH}_{4} \mathrm{Cl}$ aqueous solution $(5$ $\mathrm{mL}$ ) at $-35^{\circ} \mathrm{C}$. The biphasic mixture was stirred at room temperature for 10 minutes. The organic layer was separated and dried over $\mathrm{Na}_{2} \mathrm{SO}_{4}$. After filtration, the filtrate was purified by flash chromatography over silica gel directly (elution with $2 \%-5 \%$ ethyl acetate in hexanes) to afford homoallylic alcohol as a clear oil. 3,3'-Dibromo-1,1'-bi-2-naphthol can be easily separated and recovered by eluting silica gel column with $10 \%$ diethyl ether in benzene. 


\section{General Procedure for preparation of racemic products.}

A $15 \times 100 \mathrm{~mm}$ oven dried glass vessel was charged with stir bar and flushed with Ar. To this flask was added toluene $(1.0 \mathrm{~mL})$ and acetophenone $(44 \mu \mathrm{L}, 0.375 \mathrm{mmol})$. The mixture was stirred under $\mathrm{Ar}$ at room temperature for 5 minutes. The allyldiisopropoxyborane toluene solution $(250 \mu \mathrm{L}, 0.25 \mathrm{mmol}, 1 \mathrm{M}$ solution) was added in dropwise. The reaction mixture was stirred at room temperature for $15 \mathrm{~h}$. and quenched with saturated $\mathrm{NH}_{4} \mathrm{Cl}$ aqueous solution (1.0 $\mathrm{mL}$ ). The biphasic mixture was stirred at room temperature for 30 minutes. The organic layer was separated and dried over $\mathrm{Na}_{2} \mathrm{SO}_{4}$. After filtration, the filtrate was directly purified by flash chromatography over silica gel (elution with $2 \%-5 \%$ ethyl acetate in hexanes) to afford the desired homoallylic alcohol as a clear oil.

\section{(S)-(-)-2-Phenylpent-4-en-2-ol (11a)}<smiles>C=CCC(C)(O)c1ccccc1</smiles>

The crude mixture was purified by flash column chromatography with elution by $2 \%$ ethyl acetate in hexanes. Yield: $67 \mathrm{mg}, 83 \%$; ee: $94 \% ;[\alpha]_{\mathbf{D}}{ }^{23}=-56^{\circ}\left(\mathrm{c}=0.5, \mathrm{CHCl}_{3}\right) ; \mathrm{Lit}^{4}:[\alpha]_{\mathbf{D}}=$ $-48.8^{\circ}\left(\mathrm{c}=0.84, \mathrm{CHCl}_{3}, 82 \% \mathrm{ee}\right)$; HPLC Analysis, $\mathrm{t}_{\mathrm{r}}$ minor: 8.77min., $\mathrm{t}_{\mathrm{r}}$ major: 9.23min., [Chiralpak ${ }^{\circledR}$ AD-H column, $15 \mathrm{~cm} \times 4.6 \mathrm{~mm}$ I.D., Hexanes:IPA = 99:1, $1.0 \mathrm{~mL} / \mathrm{min}$ ]; Other sepectral data was in agreement with reported data. 4

\section{(S)-(-)-2-(3-Bromophenyl)-pent-4-en-2-ol (11b)}<smiles>C=CC[C@@](C)(O)c1cccc(Br)c1</smiles>

Reaction was run at $0.125 \mathrm{mmol}$ scale. The crude mixture was purified by flash column chromatography with elution by $2-5 \%$ ethyl acetate in hexanes. Yield: $24 \mathrm{mg}, 81 \%$ ee: $91 \%$ $[\alpha]_{\mathbf{D}}{ }^{23}=-31.7^{\circ}\left(\mathrm{c}=0.92, \mathrm{CHCl}_{3}\right)$; HPLC Analysis, $\mathrm{t}_{\mathrm{r}}$ minor: $9.6 \mathrm{~min}$., $\mathrm{t}_{\mathrm{r}}$ major: $10.7 \mathrm{~min}$, [Chiralpak ${ }^{\circledR}$ AD-H column, $15 \mathrm{~cm} \times 4.6 \mathrm{~mm}$ I.D., Hexanes:IPA = 99:1 1.0mL/min]; Other spectral data was in agreement with reported data. ${ }^{5}$

\section{(S)-(-)- 2-(4-Bromophenyl)-pent-4-en-2-ol (11c)}<smiles>C=CC[C@](C)(O)c1ccc(Br)cc1</smiles>

Reaction was run at $0.125 \mathrm{mmol}$ scale. The crude mixture was purified by flash column chromatography with elution by $2-5 \%$ ethyl acetate in hexanes. Yield: $26 \mathrm{mg}, 86 \% \quad$ ee: $99 \%$

(4) Wada, R.; Oisaki, K.; Kanai, M.; Shibasaki, M. J. Am. Chem. Soc. 2004, 126, 8910.

(5) Cunningham, A.; Mokal-Parekh, V.; Wilson, C.; Woodward, S. Org. Biomol. Chem. 2004, 2, 741. 
$[\alpha]_{\mathbf{D}}{ }^{23}=-51.1^{\circ} \quad\left(\mathrm{c}=0.23, \mathrm{CHCl}_{3}\right) ; \mathrm{Lit}^{6}:[\alpha]_{\mathbf{D}}{ }^{26}=-44^{\circ}\left(\mathrm{c}=1.6, \mathrm{CHCl}_{3}, 98 \%\right.$ ee $) ; \mathbf{H P L C}$ Analysis, $\mathrm{t}_{\mathrm{r}}$ minor: 10.9 min., $\mathrm{t}_{\mathrm{r}}$ major: $11.9 \mathrm{~min}$., [Chiralpak ${ }^{\circledR} \mathrm{AD}-\mathrm{H}$ column, $15 \mathrm{~cm} \times 4.6 \mathrm{~mm}$ I.D., Hexanes:IPA = 99:1, 1.0mL/min]; Other spectral data was in agreement with reported data. ${ }^{6}$

\section{(S)-(-)-2-(3-Trifluoromethylphenyl)-pent-4-en-2-ol (11d)}<smiles>C=CC[C@](C)(O)c1cccc(C(F)(F)F)c1</smiles>

Reaction was run at $0.125 \mathrm{mmol}$ scale. The crude mixture was purified by flash column chromatography with elution by $2-5 \%$ ethyl acetate in hexanes. Yield: $26 \mathrm{mg}, 89 \%$ ee: $91 \%$ $[\alpha]_{\mathbf{D}}{ }^{23}=-36.2^{\circ}\left(\mathrm{c}=.32, \mathrm{CHCl}_{3}\right) ; \mathrm{Lit}^{7}:[\alpha]_{\mathbf{D}}{ }^{31}=+34.3^{\circ}\left(\mathrm{c}=0.26, \mathrm{CHCl}_{3} 90 \%\right.$ ee (+)-isomer $)$; Chiral GC Analysis, $\mathrm{t}_{\mathrm{r}}$ minor: $19.4 \mathrm{~min}$., $\mathrm{t}_{\mathrm{r}}$ major: $18.8 \mathrm{~min}$., [Chiraldex $\beta$-DM column, $20 \mathrm{~m} \times$ $0.25 \mathrm{~mm}$ I.D., $\left.60-170{ }^{\circ} \mathrm{C}, 30 \mathrm{~min}\right]$; Other spectral data was in agreement with reported data. ${ }^{7}$ Absolute stereochemistry was assigned by analogy of optical rotation with 11a.

\section{(S)-(-)-2-(4-Methoxyphenyl)-4-penten-2-ol (11e)}<smiles>C=CCC(C)(O)c1ccc(OC)cc1</smiles>

Reaction was run at $0.125 \mathrm{mmol}$ scale and $.2 \mathrm{M}$ concentration. The crude mixture was purified by flash column chromatography with elution by $2-5 \%$ ethyl acetate in hexanes. Yield: 20mg, $83 \%$; ee: $>99 \%[\alpha]_{\mathbf{D}}{ }^{23}=-64.0^{\circ}\left(\mathrm{c}=, \mathrm{CHCl}_{3}\right) ; \mathrm{Lit}^{6}:[\alpha]_{\mathbf{D}}{ }^{31}=+43.1^{\circ}\left(\mathrm{c}=0.57, \mathrm{CHCl}_{3}, 79 \%\right.$ ee, (+)-isomer); HPLC Analysis, $t_{r}$ minor: 9.2 min., $t_{r}$ major: 10.1 min., [Chiralpak ${ }^{\circledR} \mathrm{OD}-\mathrm{H}$ column, $15 \mathrm{~cm} \times 4.6 \mathrm{~mm}$ I.D., Hexanes:IPA $=99: 1,1.0 \mathrm{~mL} / \mathrm{min}]$; Other spectral data was in agreement with reported data. ${ }^{7}$

\section{(S)-(-)-2-(Naphthalen-2-yl)pent-4-en-2-ol (11f)}<smiles>C=CCC(C)(O)c1ccc2ccccc2c1</smiles>

Reaction was run at $0.125 \mathrm{mmol}$ scale. The crude mixture was purified by flash column chromatography with elution by $2-5 \%$ ethyl acetate in hexanes. Yield: $21 \mathrm{mg}, 81 \%$ ee: $93 \%$ $[\alpha]_{\mathbf{D}}{ }^{23}=-60.7^{\circ}\left(\mathrm{c}=0.53, \mathrm{CHCl}_{3}\right) ; \mathrm{Lit}^{8}:[\alpha]_{\mathbf{D}}=+39.8^{\circ}\left(\mathrm{c}=0.92, \mathrm{CH}_{2} \mathrm{Cl}_{2}, 84 \%\right.$ ee, $(+)$ isomer $)$; HPLC Analysis, $\mathrm{t}_{\mathrm{r}}$ minor: 15.1 min., $\mathrm{t}_{\mathrm{r}}$ major: 16.4 min., [Chiralpak ${ }^{\circledR} \mathrm{AD}-\mathrm{H}$ column, $15 \mathrm{~cm} \times$

(6) Canales, E.; Prasad, K. G.; Soderquist, J. A. J. Am. Chem. Soc. 2005, 127, 11572.

(7) Kim, J. G.; Waltz, K. M.; Garcia, I. F.; Kwiatkowski, G. D.; Walsh, P. J. J. Am. Chem. Soc. 2004, 126, 12580.

(8) Teo, Y. -C.; Goh, J. -D.; Loh, T. -P. Org. Lett. 2005, 7, 2743. 
4.6 mm I.D., Hexanes:IPA = 99:1 1.0mL/min]; Other spectral data was in agreement with reported data. ${ }^{9}$

(S)-(-)-2-Thiophen-2-yl-pent-4-en-2-ol (11g)<smiles>C=CCC(C)(O)c1cccs1</smiles>

Reaction was run at $0.125 \mathrm{mmol}$ scale. The crude mixture was purified by flash column chromatography with elution by $2-5 \%$ ethyl acetate in hexanes. Yield: $18 \mathrm{mg}, 87 \%$ ee: $94 \%$ $[\alpha]_{\mathbf{D}}{ }^{23}=-38.3^{\circ}\left(\mathrm{c}=0.22, \mathrm{CHCl}_{3}\right)$; HPLC Analysis, $\mathrm{t}_{\mathrm{r}}$ minor: $42.2 \mathrm{~min}$., $\mathrm{t}_{\mathrm{r}}$ major: $45.0 \mathrm{~min}$, [Chiralpak ${ }^{\circledR}$ AD-H column, $25 \mathrm{~cm} \times 4.6$ mm I.D., Hexanes:IPA = 999:1 1.0mL/min]; Other spectral data was in agreement with reported data. ${ }^{9}$ Absolute stereochemistry was assigned by analogy of optical rotation with 11a.

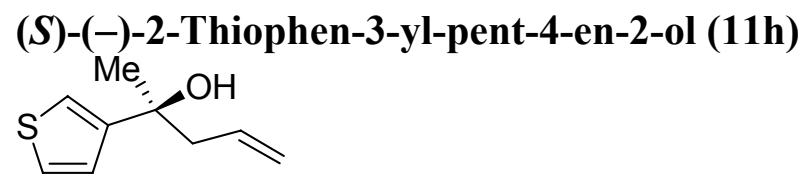

Reaction was run at $0.125 \mathrm{mmol}$ scale. The crude mixture was purified by flash column chromatography with elution by $2-5 \%$ ethyl acetate in hexanes. Yield: $18 \mathrm{mg}, 88 \%$ ee: $94 \%$ $[\alpha]_{\mathbf{D}}{ }^{23}=-46.2^{\circ}\left(\mathrm{c}=0.44, \mathrm{CHCl}_{3}\right) ; \mathrm{Lit}^{3}:[\alpha]_{\mathbf{D}}{ }^{26}=-47.1^{\circ}\left(\mathrm{c}=1.17, \mathrm{CHCl}_{3}, 94 \%\right.$ ee $) ; \mathbf{H P L C}$ Analysis, $\mathrm{t}_{\mathrm{r}}$ minor: $52.5 \mathrm{~min}$., $\mathrm{t}_{\mathrm{r}}$ major: $61.0 \mathrm{~min}$., [Chiralpak ${ }^{\circledR} \mathrm{AD}-\mathrm{H}$ column, $25 \mathrm{~cm} \times 4.6 \mathrm{~mm}$ I.D., Hexanes:IPA = 999:1 1.0mL/min]; Other spectral data was in agreement with reported data. ${ }^{5}$ Absolute stereochemistry was assigned by analogy of optical rotation with 11a.

\section{(S)-(-)-3-Phenylhex-5-en-3-ol (11i)}

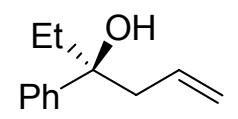

The reaction was run in $0.125 \mathrm{mmol}$ scale. The crude mixture was purified by flash column chromatography with elution by $2 \%$ ethyl acetate in hexanes. Yield: $18.5 \mathrm{mg}, 83 \%$; ee: $95 \%$; $[\alpha]_{\mathbf{D}}{ }^{23}=-24^{\circ}\left(\mathrm{c}=1.1, \mathrm{CHCl}_{3}\right) ; \mathrm{Lit}^{10}:[\alpha]_{\mathbf{D}}=-22.4^{\circ}\left(\mathrm{c}=0.71, \mathrm{CHCl}_{3}, 94 \% \mathrm{ee}\right) ;$ HPLC Analysis, $\mathrm{t}_{\mathrm{r}}$ minor: 6.23 min., $\mathrm{t}_{\mathrm{r}}$ major: $6.74 \mathrm{~min}$., [Chiralpak ${ }^{\circledR} \mathrm{AD}-\mathrm{H}$ column, $15 \mathrm{~cm} \times 4.6 \mathrm{~mm}$ I.D., Hexanes:IPA = 99:1, $1.0 \mathrm{~mL} / \mathrm{min}]$; Other spectral data was in agreement with reported data. ${ }^{4}$

\section{(S)-(-)-1-Chloro-2-phenyl-pent-4-en-2-ol (11j)}

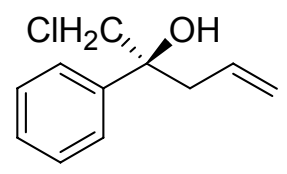

(9) Gomes, P., Gosmini, C., Perichon, J. Synthesis 2003, 12, 1909.

(10) Canales, E.; Prasad, K. G.; Soderquist, J. A. J. Am. Chem. Soc. 2005, 127, 11572. 
Reaction was run at $0.125 \mathrm{mmol}$ scale. The crude mixture was purified by flash column chromatography with elution by $2-5 \%$ ethyl acetate in hexanes. Yield: $19 \mathrm{mg}, 76 \%$ ee: $96 \%$ $[\alpha]^{23}=-18.1^{\circ}\left(c=0.44, \mathrm{CHCl}_{3}\right)$; HPLC Analysis, $\mathrm{t}_{\mathrm{r}}$ minor: $8.2 \mathrm{~min} ., 9.7 \mathrm{t}_{\mathrm{r}}$ major: min., [Chiralpak ${ }^{\circledR}$ AD-H column, $15 \mathrm{~cm} \times 4.6 \mathrm{~mm} \mathrm{I.D.,} \mathrm{Hexanes:IPA} \mathrm{=} \mathrm{99:1} \mathrm{1.0mL/min];} \mathrm{Other}$ spectral data was in agreement with reported data. ${ }^{11}$ Absolute stereochemistry was assigned by analogy of optical rotation with $\mathbf{1 1 i}$.

\section{(S)-(-)-1-Allyl-2,3-dihydro-1H-inden-1-ol (11k)}<smiles>C=CC[C@]1(O)CCc2ccccc21</smiles>

The reaction was run in $0.5 \mathrm{mmol}$ scale. The crude mixture was purified by flash column chromatography with elution by $2-5 \%$ ethyl acetate in hexanes. Yield: $76.5 \mathrm{mg}, 88 \%$; ee: $92 \%$; $[\alpha]_{\mathbf{D}}{ }^{23}=-8.8^{\circ}\left(\mathrm{c}=0.24, \mathrm{CHCl}_{3}\right) ; \mathrm{Lit}^{12}:[\alpha]_{\mathbf{D}}{ }^{31}=+6.2^{\circ}\left(\mathrm{c}=0.25, \mathrm{CHCl}_{3}, 91 \%\right.$ ee, $(+)$-isomer $) ;$ HPLC Analysis, $\mathrm{t}_{\mathrm{r}}$ major: 6.85 min., $\mathrm{t}_{\mathrm{r}}$ minor: $8.57 \mathrm{~min}$., [Chiralcel ${ }^{\circledR} \mathrm{OD}-\mathrm{H}$ column, $15 \mathrm{~cm} \times 4.6$ mm I.D., Hexanes:IPA = 98:2, $1.0 \mathrm{~mL} / \mathrm{min}$ ]; Other spectral data was in agreement with reported data. ${ }^{5}$ Absolute stereochemistry was assigned by analogy of optical rotation with $\mathbf{1 1 l}$.

\section{(S)-(-)-1-Allyl-1,2,3,4-tetrahydronaphthalen-1-ol (111)}<smiles>C=CC[C@]1(O)CCCc2ccccc21</smiles>

The reaction was run in $0.125 \mathrm{mmol}$ scale. The crude mixture was purified by flash column chromatography with elution by $2-5 \%$ ethyl acetate in hexanes. Yield: $20.7 \mathrm{mg}, 87 \%$; ee: $96 \%$; $[\alpha]_{\mathbf{D}}{ }^{23}=-41.4^{\circ}\left(\mathrm{c}=0.5, \mathrm{CHCl}_{3}\right) ; \mathrm{Lit}^{10}:[\alpha]_{\mathbf{D}}{ }^{31}=-28.5^{\circ}\left(\mathrm{c}=1.17, \mathrm{CHCl}_{3}, 84 \% \mathrm{ee}\right) ; \mathbf{H P L C}$ Analysis, $\mathrm{t}_{\mathrm{r}}$ minor: $6.56 \mathrm{~min}$., $\mathrm{t}_{\mathrm{r}}$ major: $7.40 \mathrm{~min}$., [Chiralcel ${ }^{\circledR} \mathrm{OD}-\mathrm{H}$ column, $15 \mathrm{~cm} \times 4.6 \mathrm{~mm}$ I.D., Hexanes:IPA $=98: 2,1.0 \mathrm{~mL} / \mathrm{min}]$; Other spectral data was in agreement with reported data. ${ }^{13}$

\section{(S)-(-)-4-Allyl-3,4-dihydro-2H-chromen-4-ol (11m)}<smiles>C=CC[C@]1(O)CCOc2ccccc21</smiles>

The reaction was run in $0.125 \mathrm{mmol}$ scale. The crude mixture was purified by flash column chromatography with elution by $2-5 \%$ ethyl acetate in hexanes. Yield: $19.7 \mathrm{mg}, 83 \%$; ee: $92 \%$; $[\alpha]_{\mathbf{D}}{ }^{23}=-10^{\circ}\left(\mathrm{c}=1.05, \mathrm{CHCl}_{3}\right) ; \mathrm{Lit}^{10}:[\alpha]_{\mathbf{D}}{ }^{28}=-15.8^{\circ}\left(\mathrm{c}=0.71, \mathrm{CHCl}_{3}, 92 \% \mathrm{ee}\right) ; \mathbf{H P L C}$ Analysis, $t_{r}$ minor: 8.52 min., $t_{r}$ major: 10.48 min., [Chiralcel ${ }^{\circledR}$ OD-H column, $15 \mathrm{~cm} \times 4.6 \mathrm{~mm}$

(11) Barluenga, J.; Florez, J.; Yus, M. J. Chem. Soc. Perkin Trans. I 1983, 12, 3019.

(12) Wadamoto, M.; Yamamoto, H. J. Am. Chem. Soc. 2005, 127, 14556.

(13) Casolari, S.; D’Addario, D.; Tagliavine, E. Org. Lett. 1999, 1, 1061. 
I.D., Hexanes:IPA = 98:1, $1.0 \mathrm{~mL} / \mathrm{min}]$; Other spectral data was in agreement with reported data. ${ }^{5}$ Absolute stereochemistry was assigned by analogy of optical rotation with $\mathbf{1 1 1}$.

\section{(S)-(-)-2-Cyclohexenylpent-4-en-2-ol (11n)}<smiles>C=CC[C@](C)(O)C1=CCCCC1</smiles>

The reaction was run in $0.125 \mathrm{mmol}$ scale. The crude mixture was purified by flash column chromatography with elution by $2-5 \%$ ethyl acetate in hexanes. Yield: $20 \mathrm{mg}, 91 \%$; ee: 93\%; $[\alpha]_{\mathbf{D}}{ }^{23}=-30.5^{\circ}\left(\mathrm{c}=0.75, \mathrm{CHCl}_{3}\right) ; \mathrm{Lit}^{2}:[\alpha]_{\mathbf{D}}{ }^{28}=-35.6^{\circ}\left(\mathrm{c}=1.40, \mathrm{CHCl}_{3}, 90 \% \mathrm{ee}\right)$; Chiral GC Analysis, $\mathrm{t}_{\mathrm{r}}$ minor: $18.9 \mathrm{~min}$., $\mathrm{t}_{\mathrm{r}}$ major: $19.4 \mathrm{~min}$., [Chiraldex $\beta$-DM column, $20 \mathrm{~m} \times 0.25 \mathrm{~mm}$ I.D., $\left.60-170{ }^{\circ} \mathrm{C}, 30 \mathrm{~min}\right]$; Other spectral data was in agreement with reported data. ${ }^{10}$ Absolute stereochemistry was assigned by analogy of optical rotation with 11a.

\section{(S)-(-)-1,3-Diphenylhex-5-en-1-yn-3-ol (110)}<smiles>C=CCC(O)(C#Cc1ccccc1)c1ccccc1</smiles>

The reaction was run in $0.5 \mathrm{mmol}$ scale. The crude mixture was purified by flash column chromatography with elution by 2-5\% ethyl acetate in hexanes. Yield: $115 \mathrm{mg}, 93 \%$; ee: $90 \%$; $[\alpha]_{\mathbf{D}}{ }^{23}=-5^{\circ}\left(\mathrm{c}=0.42, \mathrm{CHCl}_{3}\right)$; HPLC Analysis, $\mathrm{t}_{\mathrm{r}}$ minor: $5.63 \mathrm{~min}$., $\mathrm{t}_{\mathrm{r}}$ major: $6.33 \mathrm{~min}$, [Chiralcel ${ }^{\circledR} \mathrm{OD}-\mathrm{H}$ column, $15 \mathrm{~cm} \times 4.6 \mathrm{~mm}$ I.D., Hexanes:IPA $=95: 5,1.0 \mathrm{~mL} / \mathrm{min}$ ]; Other spectral data was in agreement with reported data. ${ }^{14}$ Absolute stereochemistry was assigned by analogy of optical rotation with 11a.

\section{(2S, 3S)-3-Methyl-2-phenylpent-4-en-2-ol (13a)}<smiles>C=CC(C)C(C)(O)c1ccccc1</smiles>

The reaction was run in $0.25 \mathrm{mmol}$ scale. The crude mixture was purified by flash column chromatography with elution by $2-5 \%$ ethyl acetate in hexanes. Yield: $30 \mathrm{mg}, 72 \%$; ee: $98 \%$; $[\alpha]_{\mathbf{D}}{ }^{23}=-67.8^{\circ}\left(\mathrm{c}=0.2, \mathrm{CHCl}_{3}\right) ; \mathrm{Lit}^{4}:[\alpha]_{\mathbf{D}}=-72.8^{\circ}\left(\mathrm{c}=1.05, \mathrm{CHCl}_{3}, 85 \% \mathrm{ee}\right) ; \mathbf{H P L C}$ Analysis, $t_{r}$ minor: 25.6 min., $t_{r}$ major: 29.80 min., [Chiralpak ${ }^{\circledR} A D-H$ column, $25 \mathrm{~cm} \times 4.6 \mathrm{~mm}$ I.D., Hexanes:IPA = 999:1, $1.0 \mathrm{~mL} / \mathrm{min}]$; Other spectral data was in agreement with reported data. ${ }^{4}$ The relative stereochemistry was determined by comparison of Chemical Shift in ${ }^{1} \mathrm{H}-\mathrm{NMR}$ with literature values. ${ }^{15}$

(14) Zheng, X.; Zhang, Y. J. Chem. Res. Synopses 2003, 8, 472.

(15) Seebach, D.; Widler, L. Helv. Chim. Acta 1982, 65, 1972. 


\section{(2S,3R)-3-Methyl-2-phenylpent-4-en-2-ol (13b)}<smiles>C=CC(C)C(C)(O)c1ccccc1</smiles>

The reaction was run in $0.25 \mathrm{mmol}$ scale. The crude mixture was purified by flash column chromatography with elution by $2-5 \%$ ethyl acetate in hexanes. Yield: $32 \mathrm{mg}, 75 \%$; ee: $98 \%$; $[\alpha]_{\mathbf{D}}{ }^{23}=+5.7^{\circ}\left(\mathrm{c}=0.25, \mathrm{CHCl}_{3}\right) ; \mathrm{Lit}^{4}:[\alpha]_{\mathrm{D}}{ }^{22}=+4.4^{\circ}\left(\mathrm{c}=1.09, \mathrm{CHCl}_{3}, 83 \% \mathrm{ee}\right) ; \mathbf{H P L C}$ Analysis, $t_{r}$ major: 26.1 min., $t_{r}$ minor: 29.0 min., [Chiralpak ${ }^{\circledR} \mathrm{AD}-\mathrm{H}$ column, $25 \mathrm{~cm} \times 4.6 \mathrm{~mm}$ I.D., Hexanes:IPA = 999:1, $1.0 \mathrm{~mL} / \mathrm{min}]$; Other sepectral data was in agreement with reported data. $^{4}$ 


\section{Nonlinear Effect Studies}

Eight $0.15 \mathrm{M}$ solution of acetophenone and $3,3^{\prime}-\mathrm{Br}_{2}$-BINOL $\mathbf{5 b}(0.015 \mathrm{M})$ of varying ee were prepared by mixing $\mathrm{CF}_{3} \mathrm{Ph}: \mathrm{CH}_{3} \mathrm{Ph}(3: 1)$ solution $(\boldsymbol{S})-\mathbf{5 b}$ and $(\boldsymbol{R})-\mathbf{5 b}$ in appropriate proportions. The ee of these catalysts solution were determined by calculation. $1 \mathrm{~mL}$ acetophenone and catalyst mixture solution was added in $15 \times 100 \mathrm{~mm}$ oven dried glass vessels equipped with stir bar under Ar. The solution was placed in $-35{ }^{\circ} \mathrm{C}$ bath and allyldiisopropylborane $(100 \mu \mathrm{L}, 1 \mathrm{M}$ toluene solution) was added. The reaction mixture was stirred at $-35{ }^{\circ} \mathrm{C}$ for 15 hours and quenched with ammonium chloride aqueous solution $(1 \mathrm{~mL})$. The biphasic mixture was stirred at room temperature for $15 \mathrm{~min}$. The organic layer was separated and run through a anhydrous $\mathrm{Na}_{2} \mathrm{SO}_{4}$ plug. The solution was purified by flash column chromatography on $\mathrm{SiO}_{2}$ directly without concentration. The isolated products were analalyzed by chiral HPLC (Chiralpak ${ }^{\circledR}$ AD-H $25 \mathrm{~cm} \times 4.6 \mathrm{~mm}, 1 \mathrm{~mL} / \mathrm{min}, 999: 1$ hexanes: $i-\mathrm{PrOH})$ to determine the ee. Similar $(+)-\mathrm{NLE}$ and yield trend were reported for BINOL-Ti catalyst. ${ }^{16}$

Table S1. Nonlinear effect of the asymmetric allylboration reaction.

\begin{tabular}{ccc}
\hline Catalyst $\mathbf{5 b}$ ee $(\%)$ & Yield of 11a $(\%)$ & Product 11a ee $(\%)^{a}$ \\
\hline 0 & 6 & 1 \\
10 & 14 & 35 \\
25 & 22 & 64 \\
35 & 35 & 69 \\
50 & 47 & 83 \\
60 & 56 & 90 \\
75 & 64 & 91 \\
100 & 83 & 90
\end{tabular}

Figure S1. Positive non-linear effect in allylboration of acetophenone

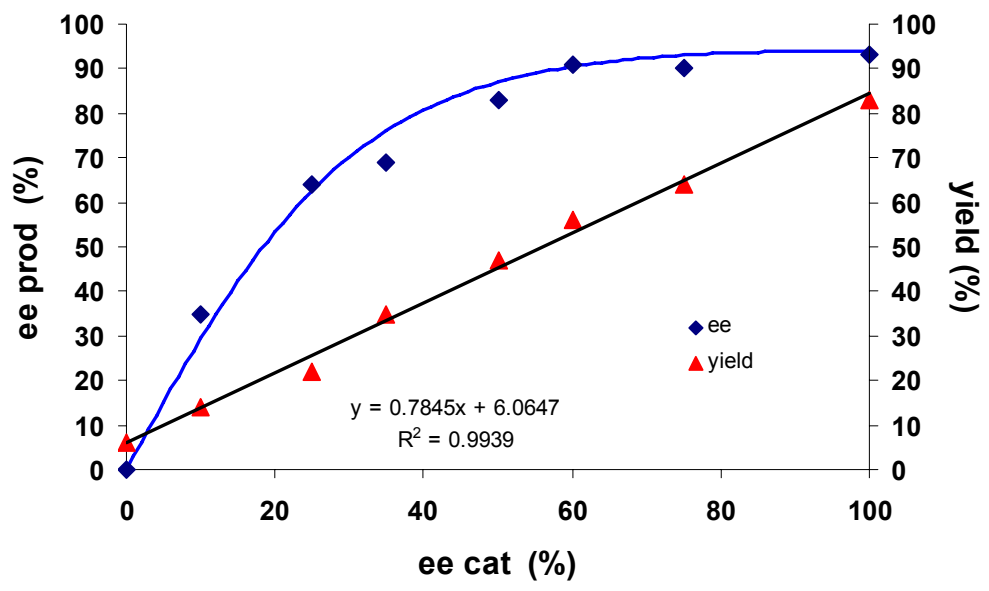

(16) Mikami, K.; Matsumoto, Y. Tetrahedron 2004, 60, 7715. 


\section{Kinetic Experiments}

General experimental to determine catalyst order. A $5 \mathrm{~mL}$ vial equipped with a stir bar was charged with $(S)-3,3$ '- $-\mathrm{Br}_{2}$-BINOL (11 mg, $0.025 \mathrm{mmol}, 10 \mathrm{~mol} \%$ catalyst loading) and toluene $(1 \mathrm{~mL})$. The vial was sealed with a septum and equipped with the DiComp ReactIR 4000 Probe. The solution was cooled at $0{ }^{\circ} \mathrm{C}$ for $10 \mathrm{~min}$ and background was taken at this moment. To this catalyst solution was added acetophenone $(30 \mu \mathrm{L}, 0.25 \mathrm{mmol})$ and stirred for $5 \mathrm{~min}$. Allyldiisopropoxyborane $(90 \mu \mathrm{L}, 0.50 \mathrm{mmol})$ was added. The solution was stirred at $0{ }^{\circ} \mathrm{C}$ for 120 min and the carbonyl stretch of acetophenone $\mathbf{1 0 a}$ was monitored at $1690.27 \mathrm{~cm}^{-1}$ by ReactIR at real time.

Table S2. Effect of initial $(S)-3,3$ '- $\mathrm{Br}_{2}-\mathrm{BINOL}$ concentration on the rate of allylboration reaction.

\begin{tabular}{cc}
\hline$[(\boldsymbol{S})-\mathbf{5 b}]_{\text {initial }}(\mathrm{M})$ & $\mathrm{k}_{\mathrm{obs}}(\mathrm{mM} / \mathrm{min})$ \\
\hline 0.00625 & 0.37 \\
0.0125 & 1.06 \\
0.025 & 2.12 \\
0.0375 & 3.50
\end{tabular}

Figure S2. First order of catalyst in allylboration of acetophenone

First Order of Catalyst

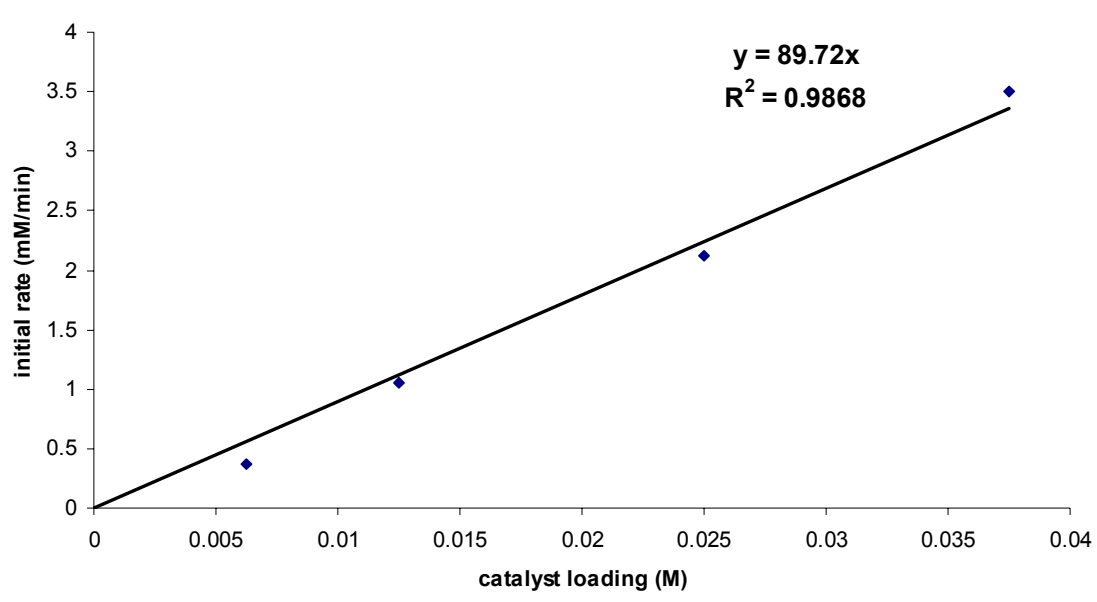




\section{NMR Experiments:}

\section{$\underline{{ }^{1} \text { H-NMR of authentic samples }}$}

To an a dry NMR tube purged with $\mathrm{Ar}$, a pure sample and $\mathrm{CD}_{3} \mathrm{C}_{6} \mathrm{D}_{5}(1.0 \mathrm{~mL})$ were added. The ${ }^{1} \mathrm{H}-\mathrm{NMR}$ was taken at room temperature. Three single peaks (7.10 ppm, $7.02 \mathrm{ppm}, 6.98 \mathrm{ppm}$ ) were used as reference peaks in $\mathrm{CD}_{3} \mathrm{C}_{6} \mathrm{D}_{5}$. The impurity in allyldiisopropoxyborane was allylboronic acid due to the slight hydrolysis. ${ }^{17}$ In ${ }^{1} \mathrm{H}-\mathrm{NMR}$ of monomethyl protected 3,3'- $\mathrm{Br}_{2}$ BINOL 8, the peak at $7.95 \mathrm{ppm}$ was assigned to $\mathrm{H}_{\mathrm{a}}$ based on the chemical shift caused by methyl protection. ${ }^{18}$
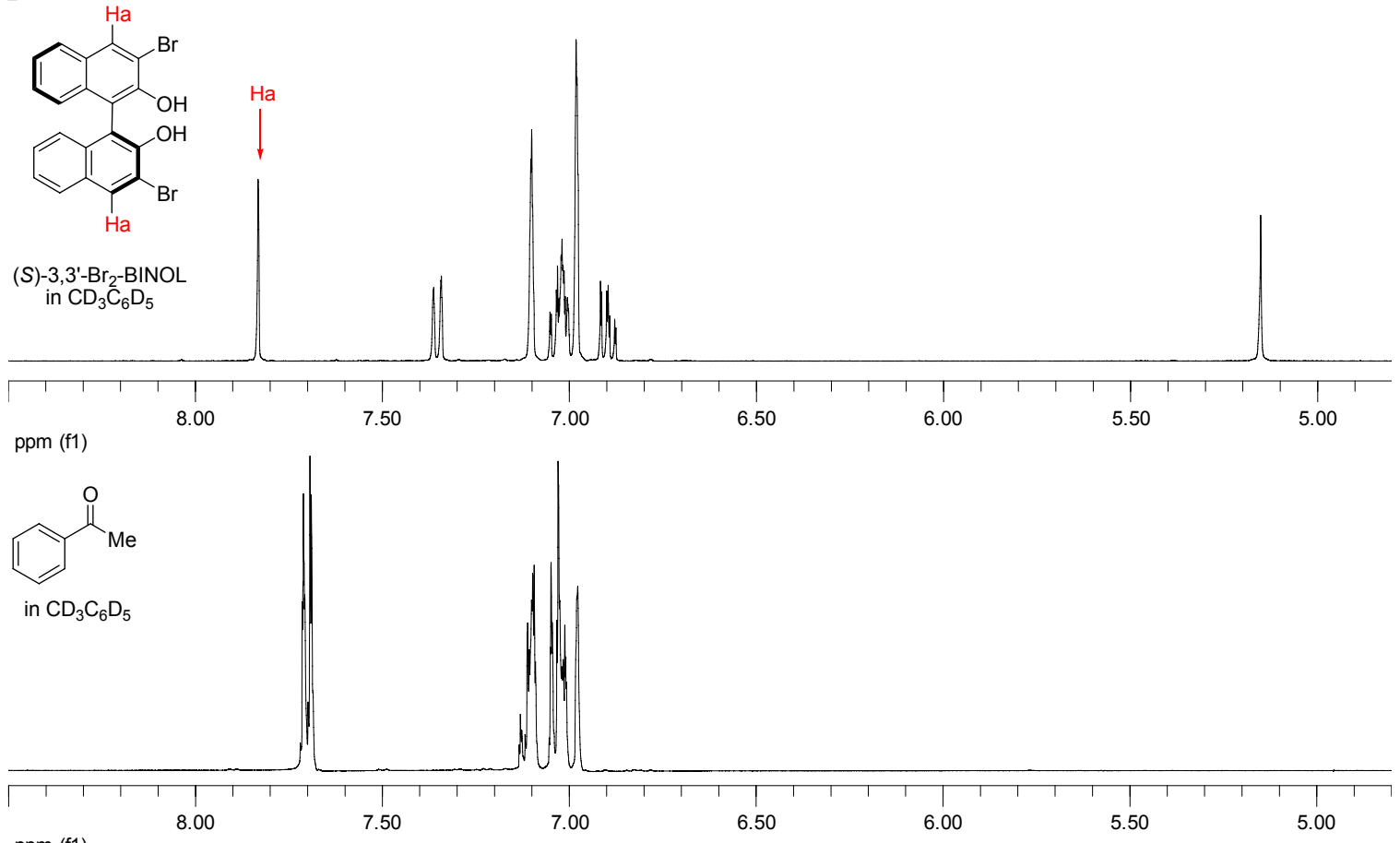

ppm (f1)
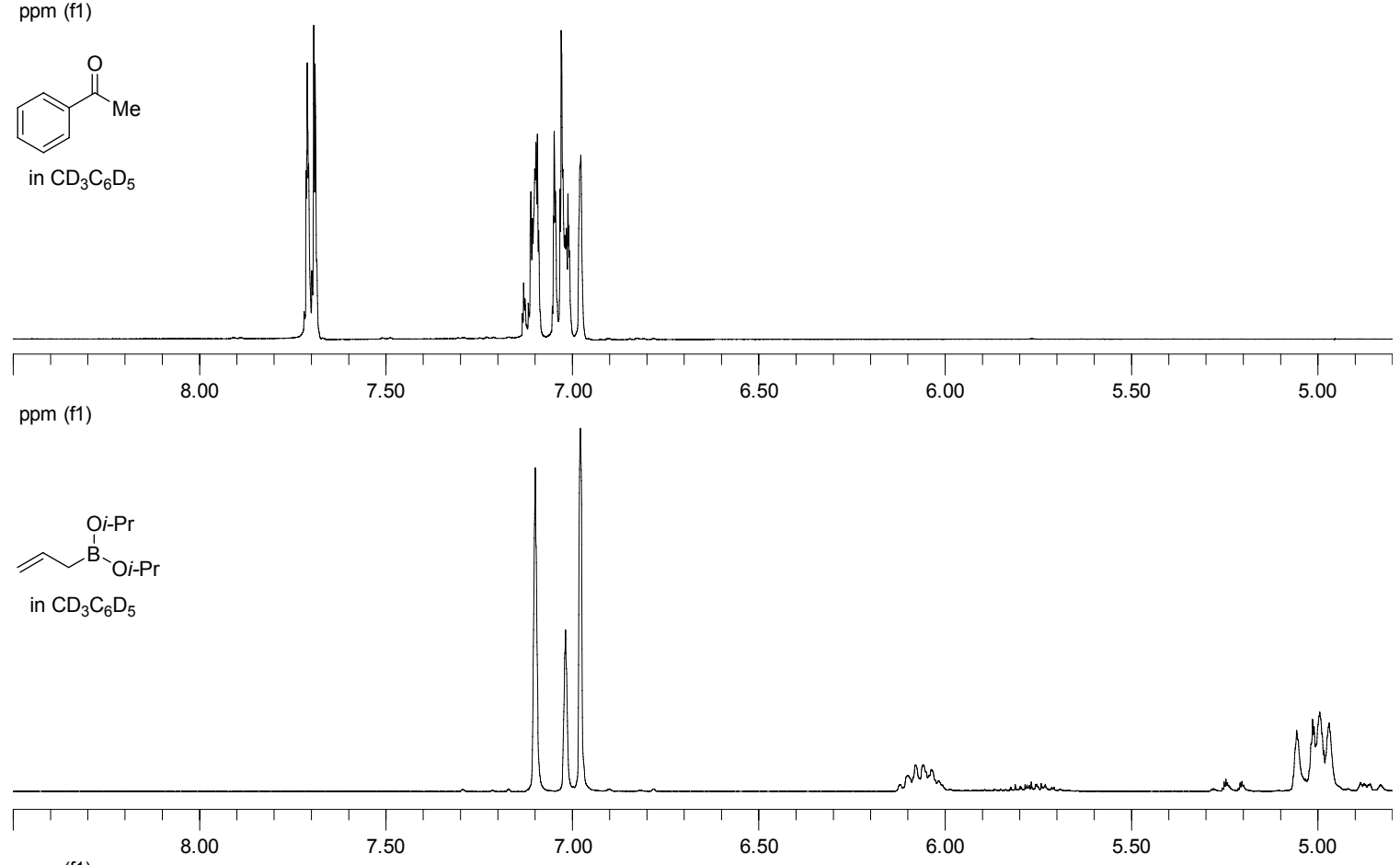

ppm (f1)

(17) $4 \AA$ molecular sieves as additive was proved to diminish the enantioselectivity in the addition of allyldiisopropoxyborane to acetophenone.

(18) Orasawara, K.; Takahashi, M. Tetrahedron: Asymmetry 1997, 8, 3125. 


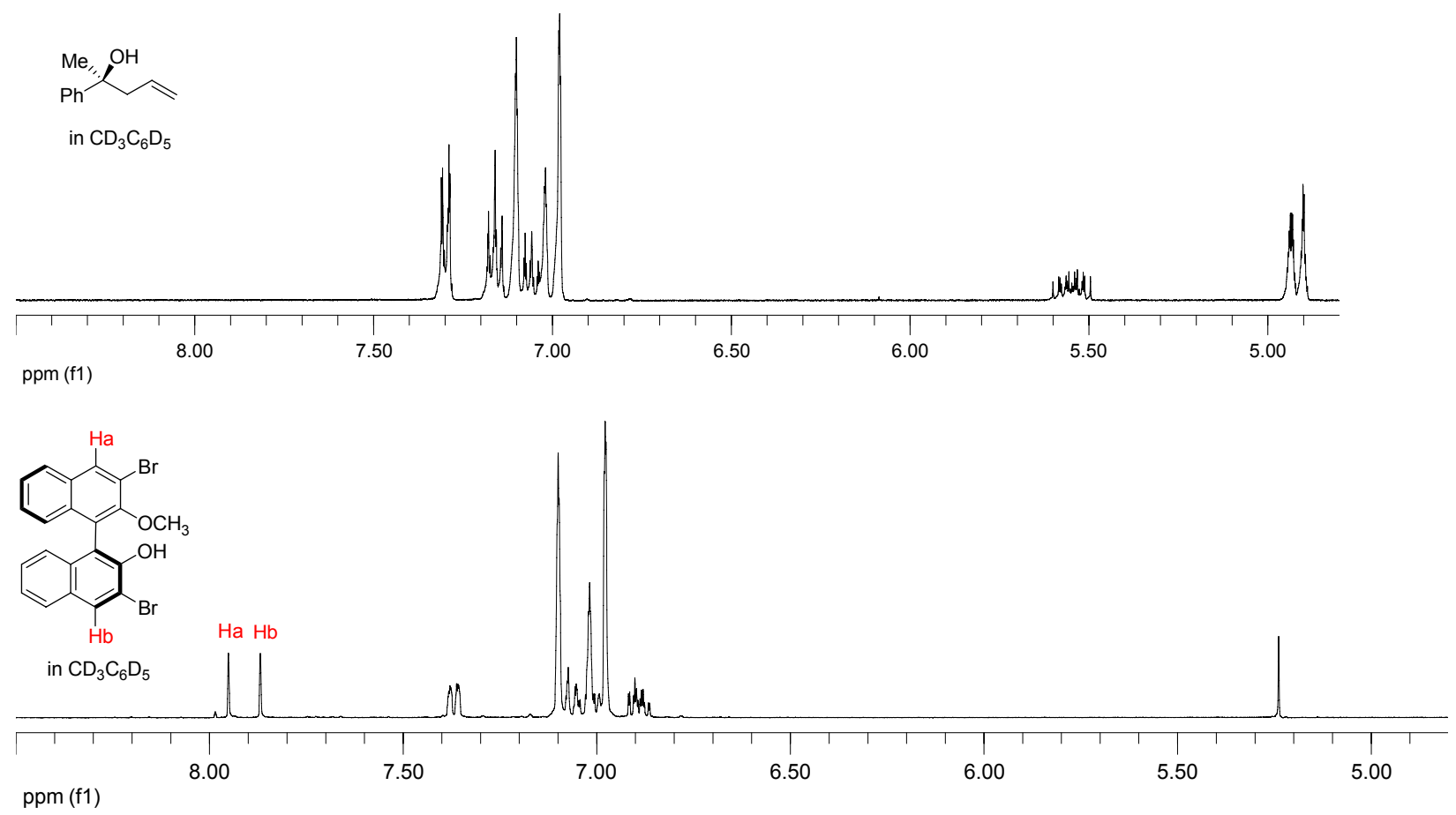

Exchange of one isopropoxy ligand of boronate 10 with $3,3^{\prime}-\mathrm{Br}_{2}-\mathrm{BINOL}$

To a dry NMR tube, allyldiisopropoxyborane $(40 \mathrm{uL}, 0.10 \mathrm{mmol})$ and $\mathrm{CD}_{3} \mathrm{C}_{6} \mathrm{D}_{5}(1.0 \mathrm{~mL})$ were added under Ar. 3,3'- $\mathrm{Br}_{2}$-BINOL $(6.8 \mathrm{mg}, 0.015 \mathrm{mmol})$ was added. The solution was left under $\mathrm{Ar}$ at room temperature for 20 hours and an ${ }^{1} \mathrm{H}-\mathrm{NMR}$ was taken. Dissymmetrical chemical shifts of $3,3^{\prime}-\mathrm{Br}_{2}$-BINOL aromatic proton were observed. We attribute this differentiation to the exchange of one boronate isopropxy ligand with 3,3 ' $-\mathrm{Br}_{2}$-BINOL as indicated in the following structure. Peak at $7.97 \mathrm{ppm}$ was assigned to $\mathrm{H}_{\mathrm{a}}$ according the reported chemical shift of BINOL boronate ${ }^{19}$ and comparison of the ${ }^{1} \mathrm{H}-\mathrm{NMR}$ of $3,3^{\prime}-\mathrm{Br}_{2}$-BINOL mono-methyl ether $\mathbf{8}$. The further downfield chemical shift of $\mathrm{H}_{\mathrm{a}}$ than $\mathrm{H}_{\mathrm{b}}$ is presumably due to the bonding of BINOL oxygen to boron. Peak at $7.94 \mathrm{ppm}$ was assigned to $\mathrm{H}_{\mathrm{b}}$ and the downfield chemical shift is probably caused by H-bonding to the boronate isopropoxy ligand.

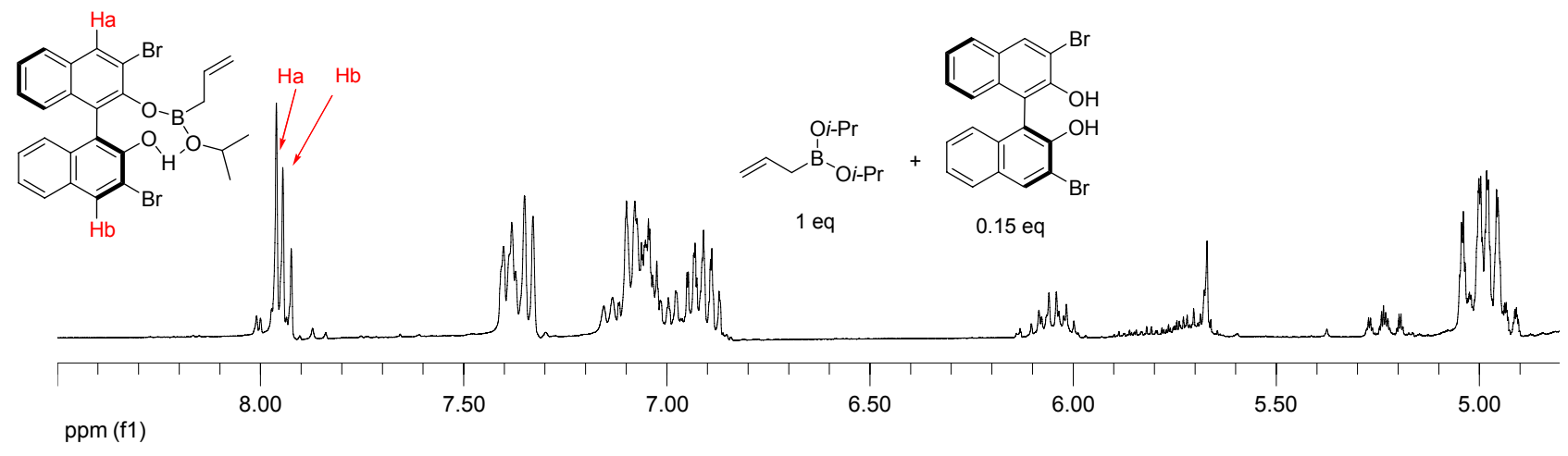

(19) Thormeier, S.; Carboni, B.; Kaufmann, D. E. Journal of Organometallic Chemistry 2002, 657, 136 


\section{Acceleration effect of external alcohol in the formation of $3,3^{\prime}$-Br 2 -BINOL boronate complex and the allylboration of ketones.}

To a dry NMR tube, allyldiisopropoxyborane $(40 \mathrm{uL}, 0.10 \mathrm{mmol})$ and $\mathrm{CD}_{3} \mathrm{C}_{6} \mathrm{D}_{5}(1.0 \mathrm{~mL})$ were added under Ar. 3, 3' $-\mathrm{Br}_{2}$-BINOL $(6.8 \mathrm{mg}, 0.015 \mathrm{mmol})$ and $t$-BuOH $(2 \mathrm{uL}, 0.015 \mathrm{mmol})$ were subsequently added. The solution was left under Ar at room temperature for $30 \mathrm{~min}$ and an ${ }^{1} \mathrm{H}-$ NMR was taken. $60 \%$ of $3,3^{\prime}-\mathrm{Br}_{2}$-BINOL had been converted to desired BINOL boronate complex according to the integrations of $\mathrm{H}_{\mathrm{a}}, \mathrm{H}_{\mathrm{b}}$, and $\mathrm{H}_{\mathrm{c}}$. Without $t-\mathrm{BuOH}$, only $10 \%$ of BINOL boronate complex formation was observed after $30 \mathrm{~min}$. This experiment indicates the external alcohol accelerates the BINOL boronate complex formation.

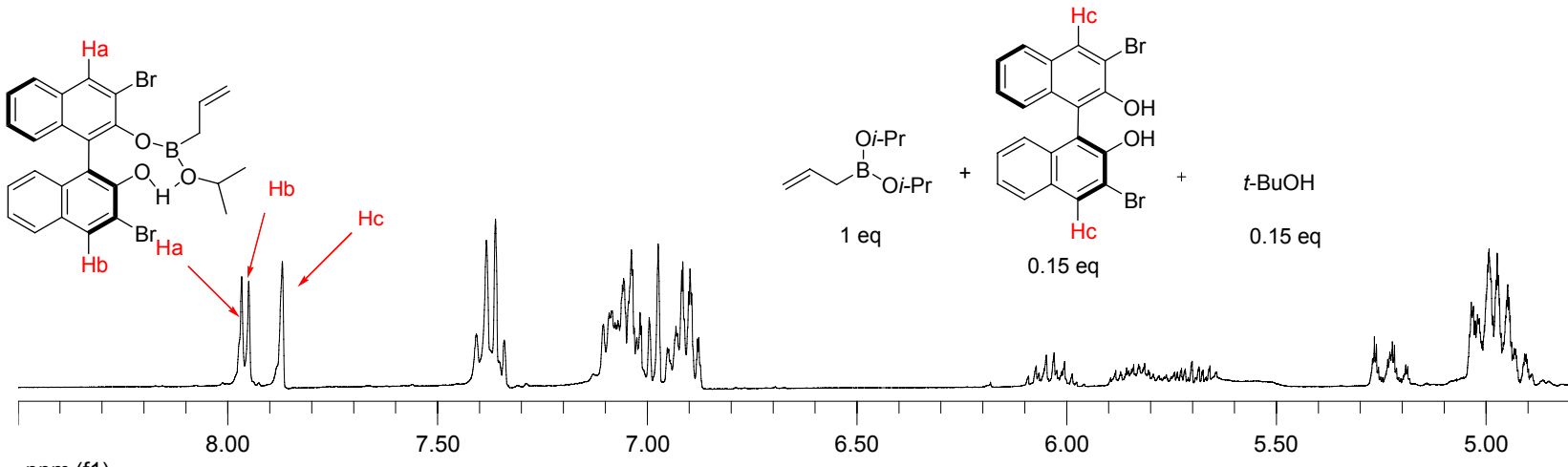

$\operatorname{ppm}(\mathrm{f} 1)$

To a dry NMR tube, allyldiisopropoxyborane $(40 \mathrm{uL}, 0.10 \mathrm{mmol})$ and $\mathrm{CD}_{3} \mathrm{C}_{6} \mathrm{D}_{5}(1.0 \mathrm{~mL})$ were added under Ar. 3, 3'- $-\mathrm{Br}_{2}$-BINOL (6.8 mg, $\left.0.015 \mathrm{mmol}\right), t$-BuOH (2 uL, $\left.0.015 \mathrm{mmol}\right)$ and acetophenone (33 uL, $0.015 \mathrm{mmol})$ were subsequently added. The solution was left under Ar at room temperature and a ${ }^{1} \mathrm{H}-\mathrm{NMR}$ was taken. $60 \%$ conversion to the product was observed after 30 min according to the integrations of $\mathrm{H}_{\mathrm{d}}$ and $\mathrm{H}_{\mathrm{e}}$. Without $t$-BuOH , only $25 \%$ conversion to the product was observed. This experiment indicates the external alcohol help the turn over of the catalyst.

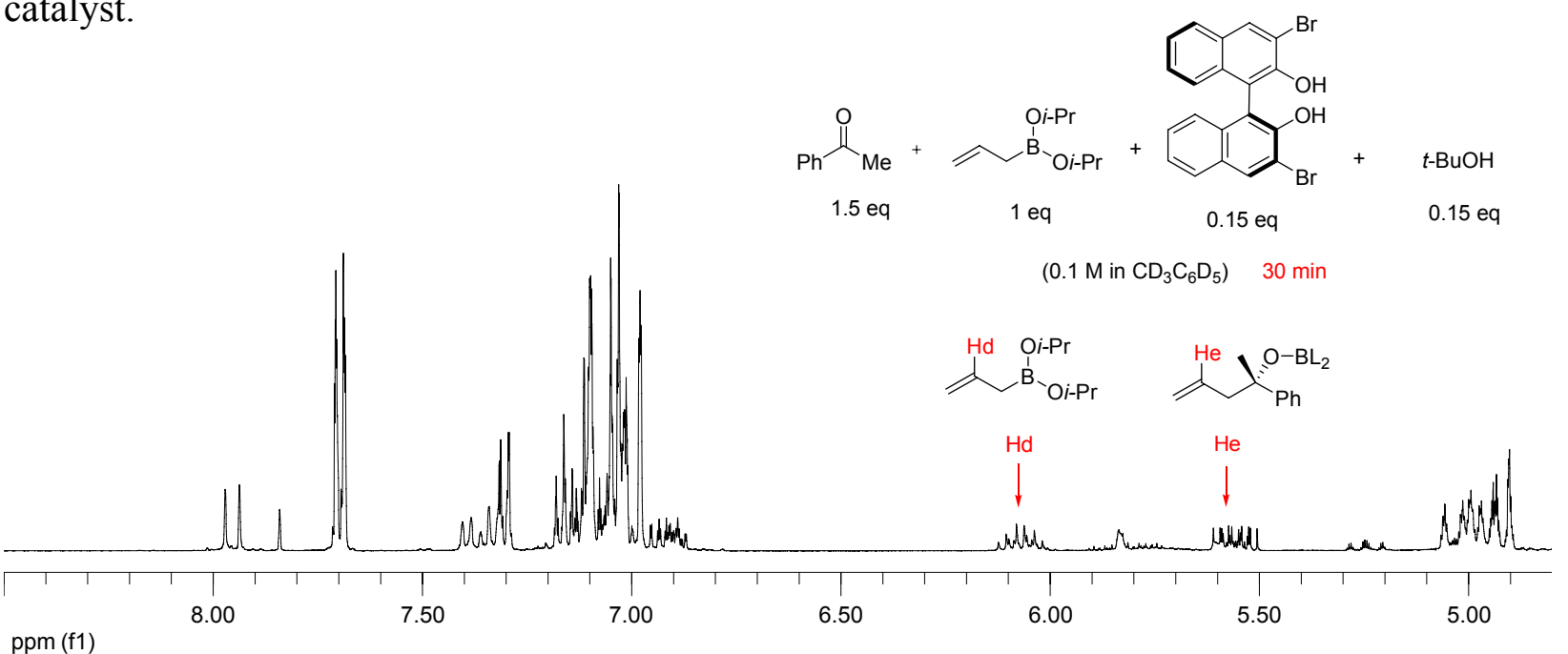




\section{Monitoring allylboration of acetophenone by ${ }^{1} \mathrm{H}-\mathrm{NMR}$}

To a dry NMR tube, acetophenone $(33 \mathrm{uL}, 0.015 \mathrm{mmol})$ and $\mathrm{CD}_{3} \mathrm{C}_{6} \mathrm{D}_{5}(1.0 \mathrm{~mL})$ were added under Ar. 3, 3'- $-\mathrm{Br}_{2}$-BINOL (6.8 mg, $\left.0.015 \mathrm{mmol}\right)$ was added and the solution was shaken for 2 min until it became clear. Allyldiisopropoxyborane $(40 \mathrm{uL}, 0.10 \mathrm{mmol})$ was subsequently added. The reaction mixture was monitored by ${ }^{1} \mathrm{H}-\mathrm{NMR}$ at room temperature. ${ }^{1} \mathrm{H}-\mathrm{NMR}$ was taken at 30 min, $60 \mathrm{~min}, 120 \mathrm{~min}$ and 20 hours. The 4,4'-proton, $\mathrm{H}_{\mathrm{a}}$ and $\mathrm{H}_{\mathrm{b}}$, differentiation of $\mathbf{5 b}$ was observed immediately after addition of allyldisopropoxyborane. We attribute this differentiation to the exchange of one boronate isopropoxy ligand with one BINOL $\mathbf{5 b}$ alcohol. $80 \%$ of $\mathbf{5 b}$ boronate complex was formed after 2 hours and maintained during the course of the reaction.

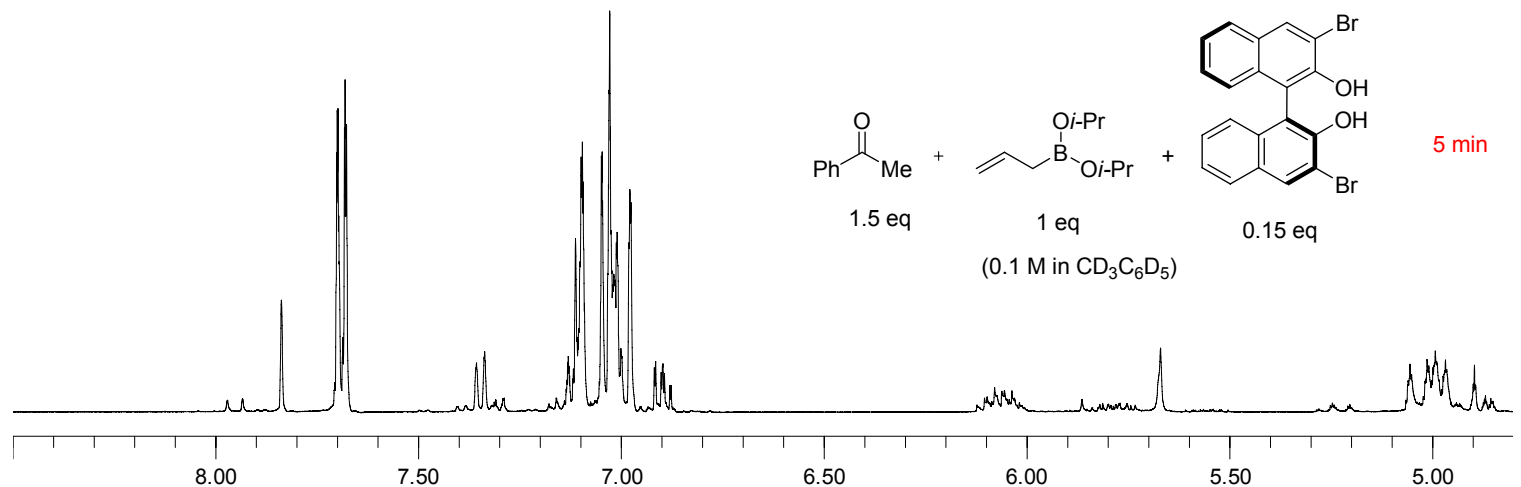
ppm (f1)

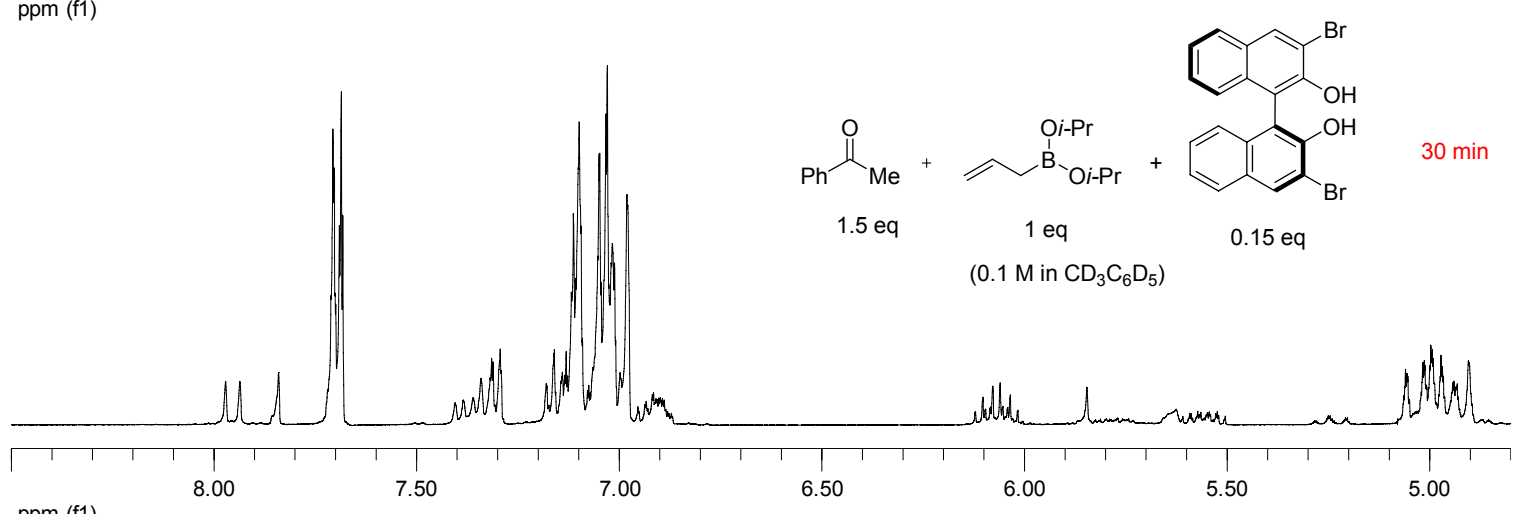
ppm (f1)
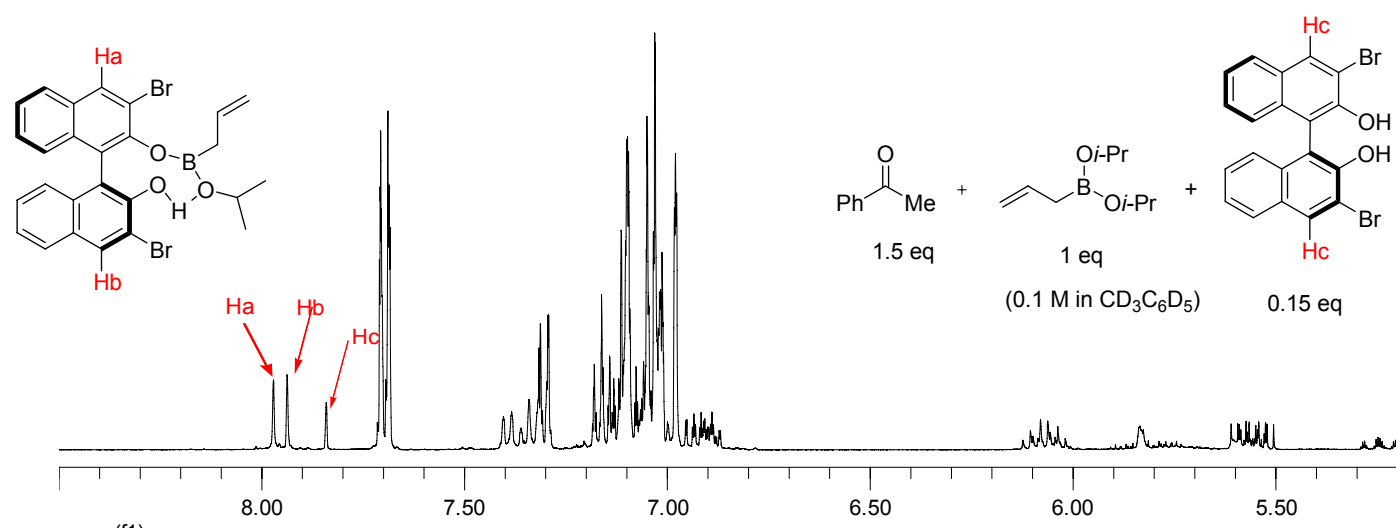

$120 \mathrm{~min}$

ppm (f1) 


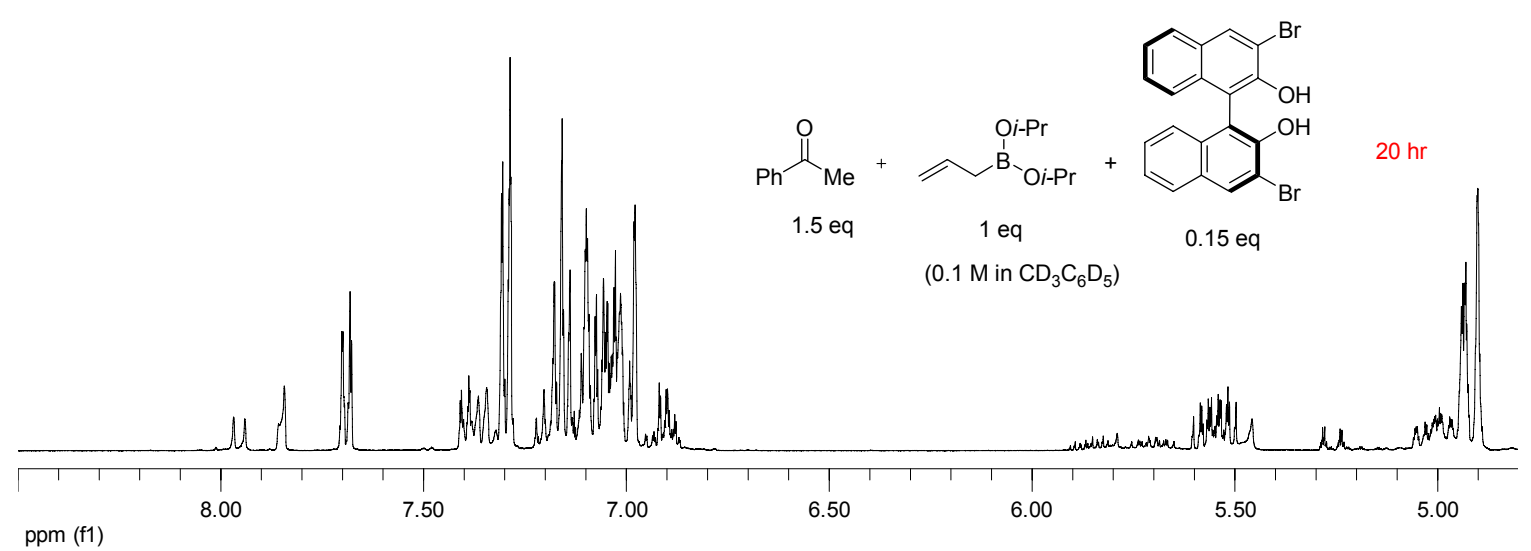


Table 2, entry 1: 11a Chiralpak ${ }^{\circledR}$ AD-H Column, Hexane:IPA = 99:1, $1.0 \mathrm{~mL} / \mathrm{min}, 214 \mathrm{~nm}$

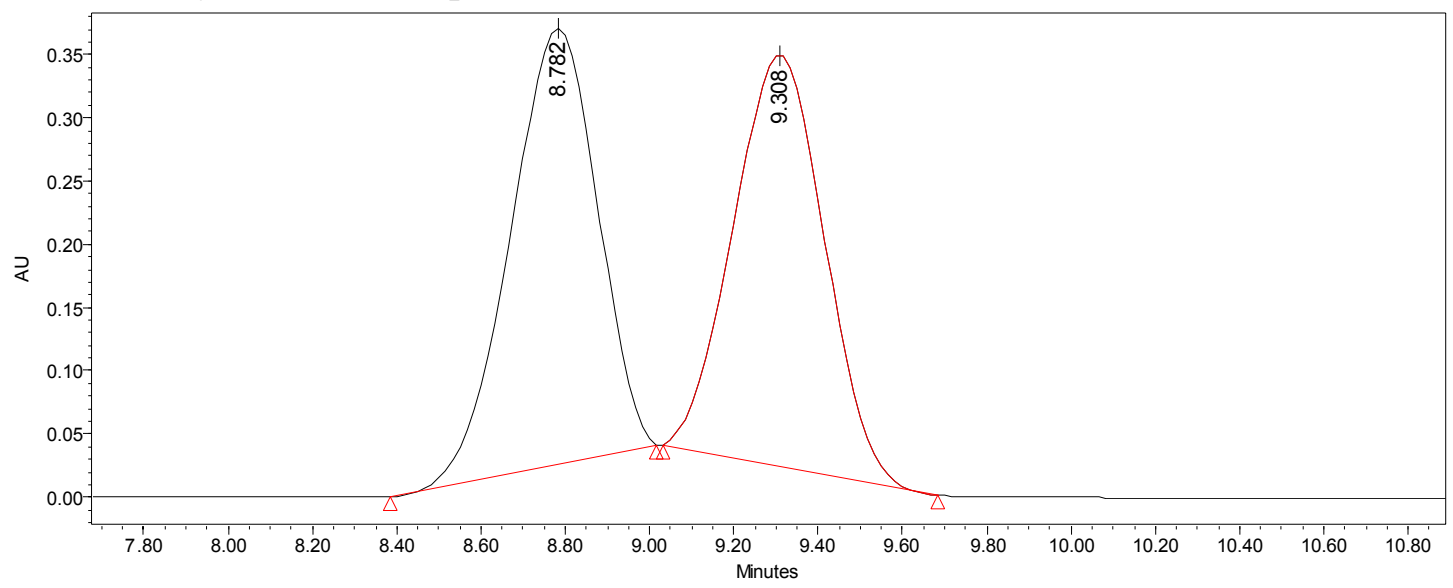

\begin{tabular}{|r|r|r|r|}
\hline & Retention Time & Area & \% Area \\
\hline 1 & 8.782 & 4918989 & 50.14 \\
\hline 2 & 9.308 & 4892440 & 49.86 \\
\hline
\end{tabular}
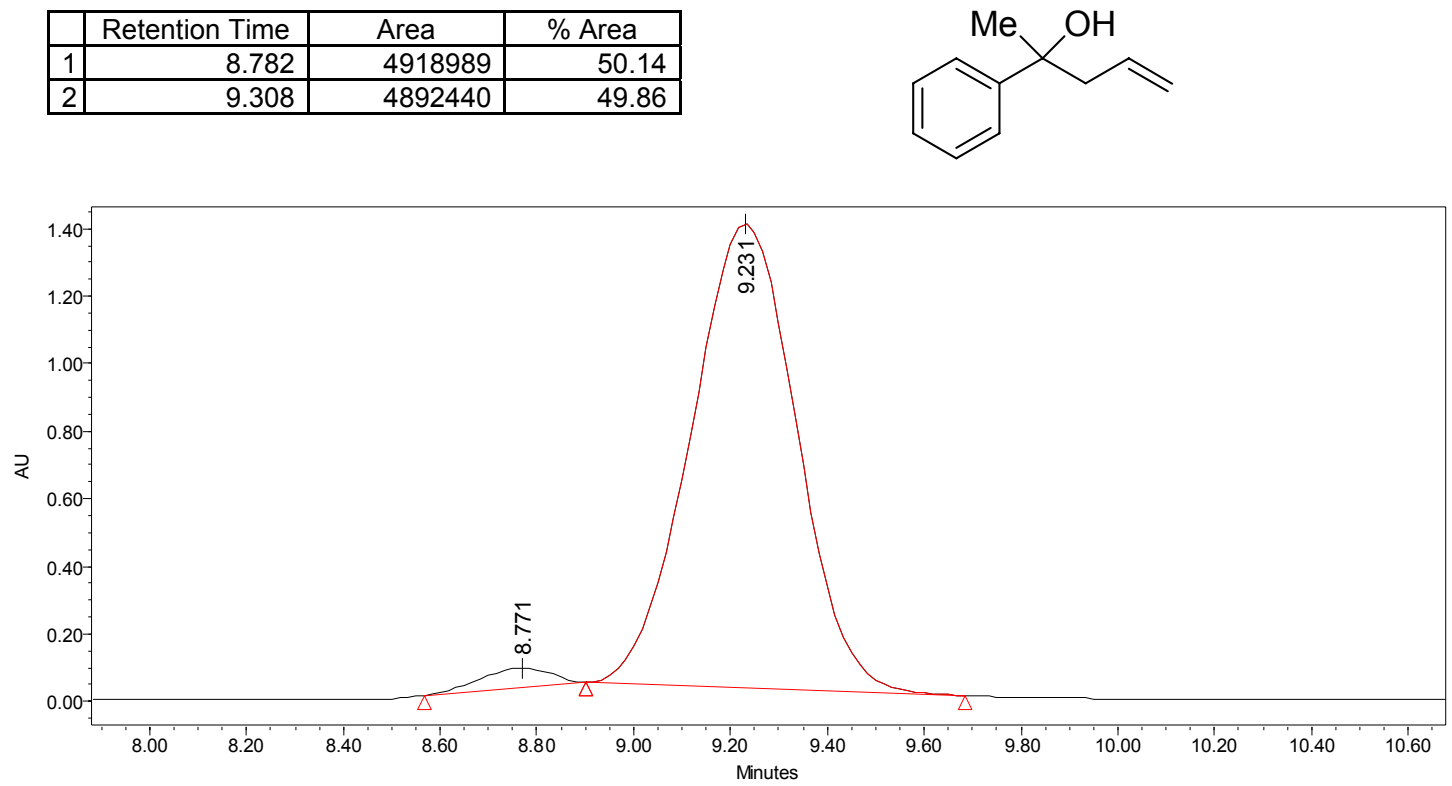

\begin{tabular}{|r|r|r|r|}
\hline & Retention Time & Area & \% Area \\
\hline 1 & 8.771 & 607070 & 2.83 \\
\hline 2 & 9.231 & 20852446 & 97.17 \\
\hline
\end{tabular}<smiles>C=CC[C@@](C)(O)c1ccccc1</smiles> 
Table 2, entry 2: 11b Chiralpak ${ }^{\circledR}$ AD-H Column, Hexane:IPA = 99:1, $1.0 \mathrm{~mL} / \mathrm{min}, 214 \mathrm{~nm}$

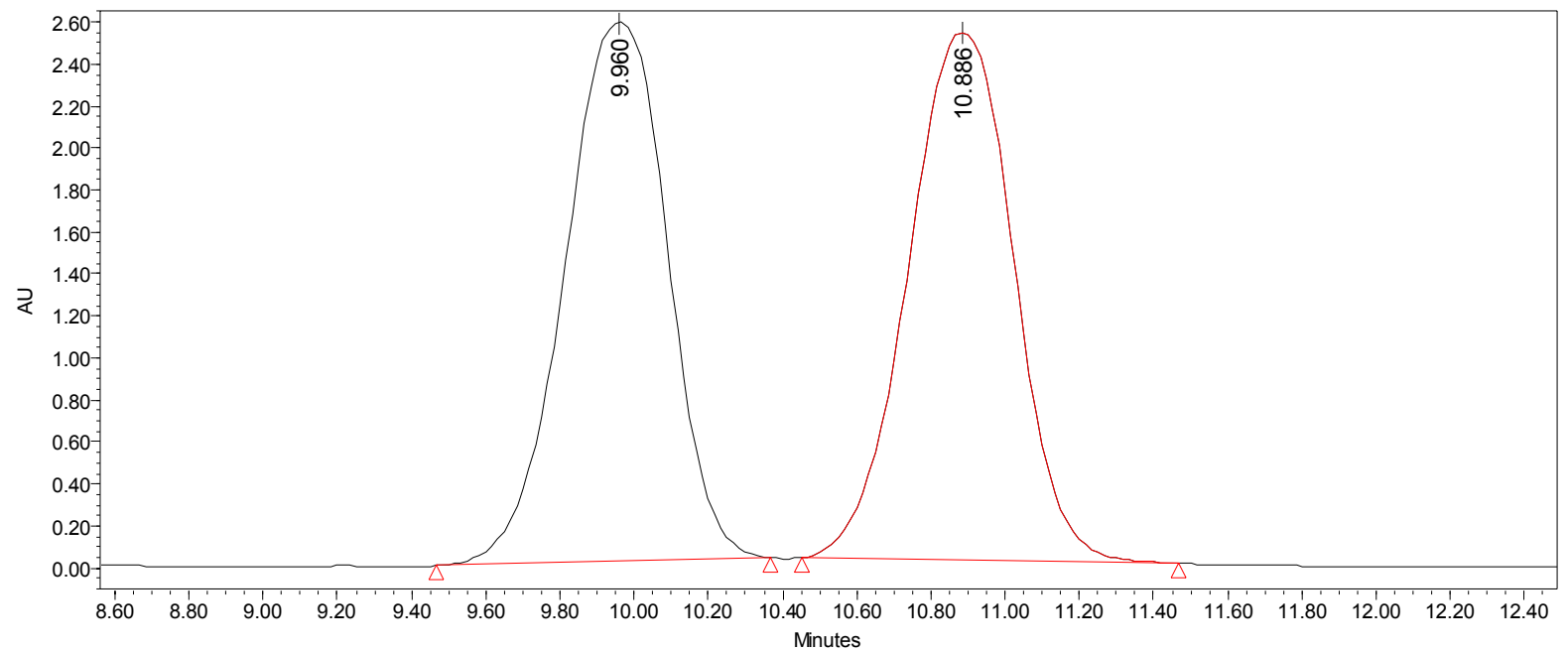

\begin{tabular}{|r|r|r|r|}
\hline & Retention Time & Area & \% Area \\
\hline 1 & 9.960 & 49096258 & 49.69 \\
\hline 2 & 10.886 & 49714575 & 50.31 \\
\hline
\end{tabular}

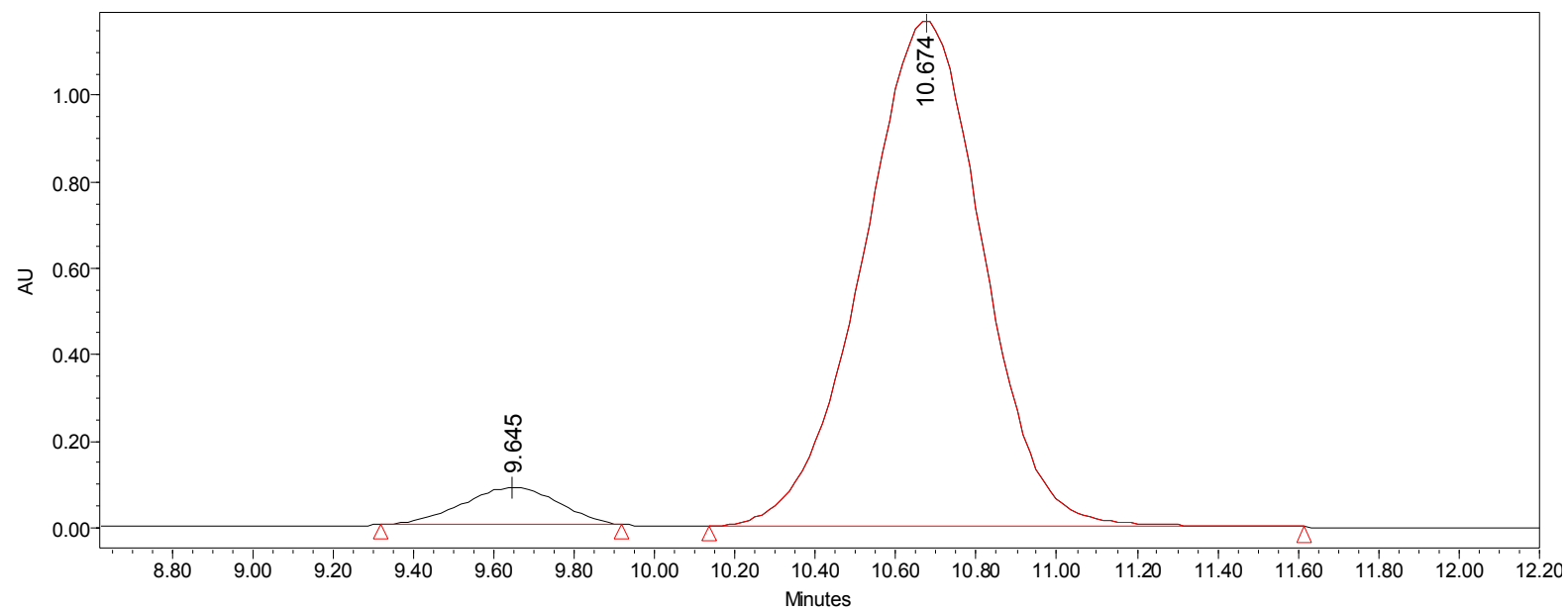

\begin{tabular}{|r|r|r|r|}
\hline & Retention Time & Area & \% Area \\
\hline 1 & 9.645 & 1418275 & 4.51 \\
\hline 2 & 10.674 & 30029064 & 95.49 \\
\hline
\end{tabular}<smiles>C=CCC(C)(O)c1cccc(Br)c1</smiles> 
Table 2, entry 3: 11c Chiralpak ${ }^{\circledR}$ AD-H Column, Hexane:IPA = 99:1, $1.0 \mathrm{~mL} / \mathrm{min}, 214 \mathrm{~nm}$

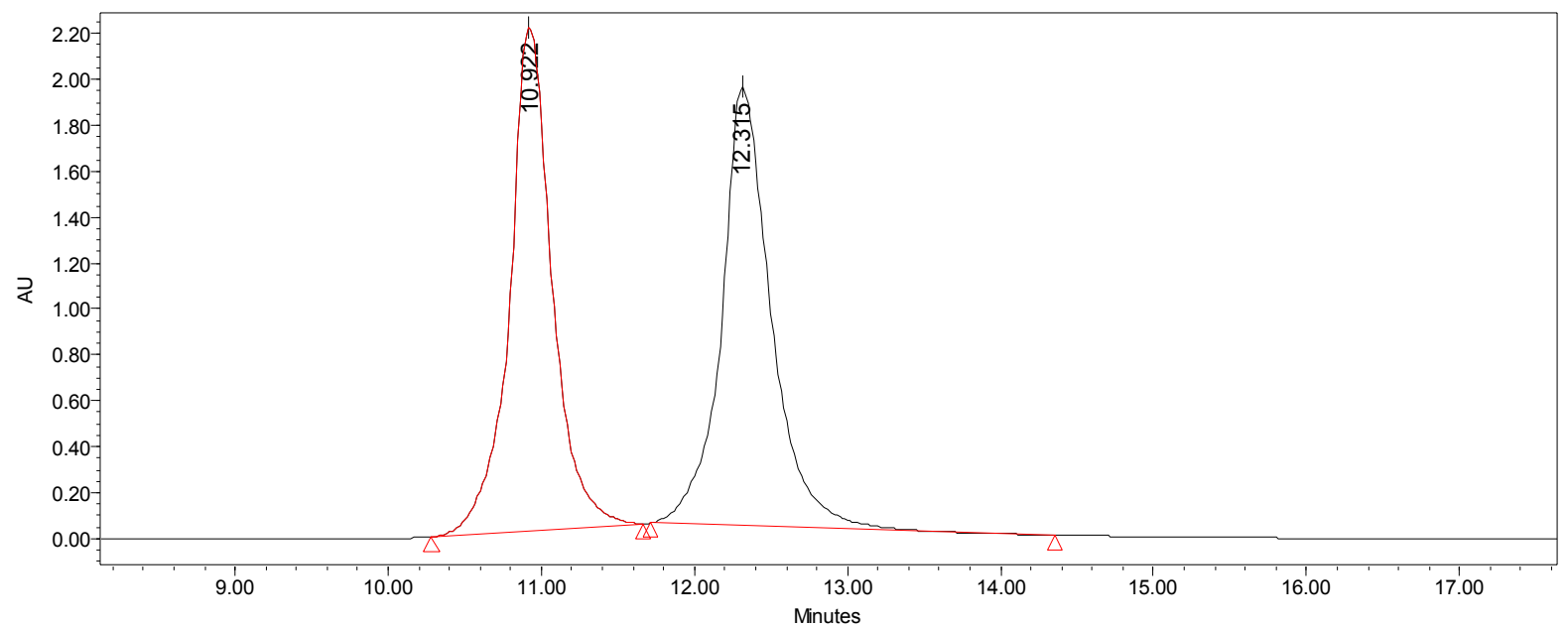

\begin{tabular}{|l|r|c|r|}
\hline & Retention Time & Area & \% Area \\
\hline 1 & 10.922 & 42537363 & 49.57 \\
\hline 2 & 12.315 & 43282435 & 50.43 \\
\hline
\end{tabular}
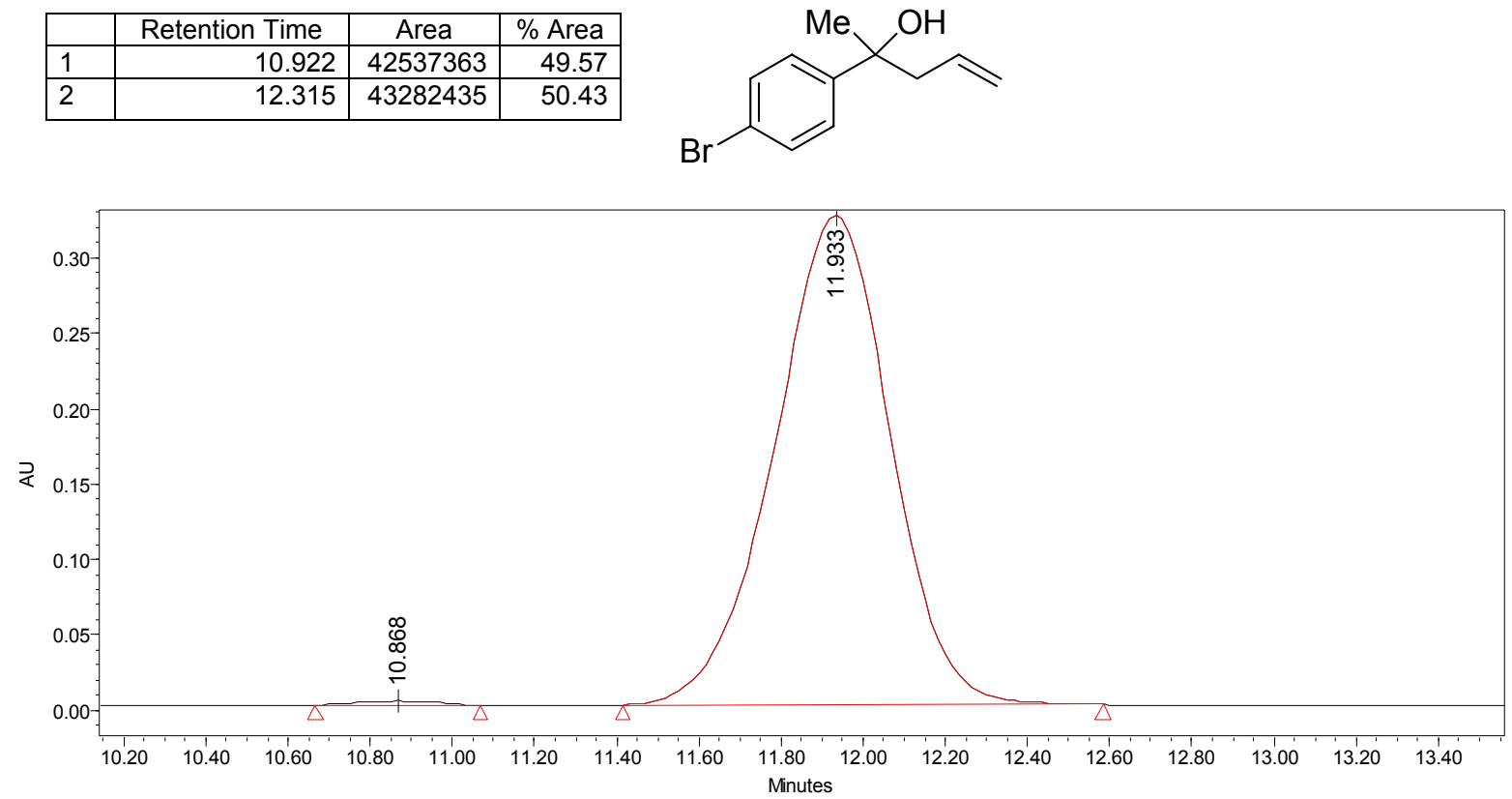

\begin{tabular}{|l|r|r|r|}
\hline & Retention Time & \multicolumn{1}{c|}{ Area } & \% Area \\
\hline 1 & 10.868 & 34495 & 0.54 \\
\hline 2 & 11.933 & 6360294 & 99.46 \\
\hline
\end{tabular}<smiles>C=CC[C@@](C)(O)c1ccc(Br)cc1</smiles> 
Table 2, entry 4: 11d ChiralGC, Chiraldex B-DM column, $30 \mathrm{~m} \times 0.25 \mathrm{~mm}$ I.D., $60-170^{\circ} \mathrm{C}$, 30min, FID
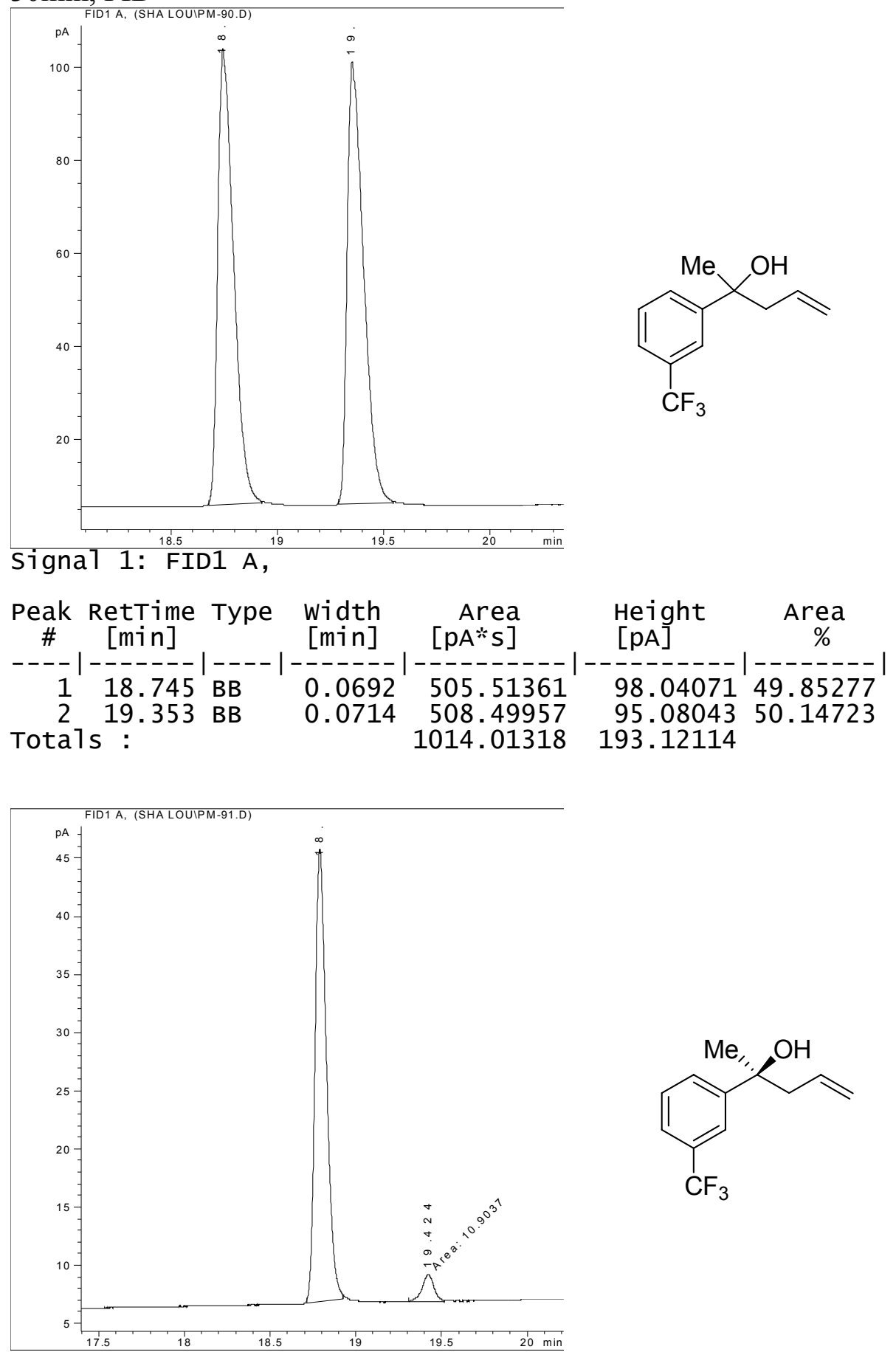

Signal 1: FID1 A,

\begin{tabular}{|c|c|c|c|c|c|c|}
\hline$\underset{\#}{\text { Peak }}$ & $\begin{array}{c}\text { RetTime } \\
{[\text { min] }}\end{array}$ & Type & $\begin{array}{l}\text { Width } \\
\text { [min] }\end{array}$ & $\begin{array}{r}\text { Area } \\
{\left[\mathrm{pA}^{*} \mathrm{~s}\right]}\end{array}$ & $\begin{array}{l}\text { Height } \\
{[\mathrm{pA}]}\end{array}$ & $\begin{array}{c}\text { Area } \\
\%\end{array}$ \\
\hline $\begin{array}{r}1 \\
2 \\
\text { Tota1 }\end{array}$ & $\begin{array}{l}18.791 \\
19.423 \\
\text { ls : }\end{array}$ & MM & $\begin{array}{l}0.0813 \\
0.0706\end{array}$ & $\begin{array}{r}191.96854 \\
9.28314 \\
201.25167\end{array}$ & $\begin{array}{r}39.37783 \\
2.19145 \\
41.56928\end{array}$ & $\begin{array}{r}95.38730 \\
4.61270\end{array}$ \\
\hline
\end{tabular}


Table 2, entry 5: 11e Chiralcel ${ }^{\circledR}$ OD-H Column, Hexane:IPA $=99: 1,1.0 \mathrm{~mL} / \mathrm{min}, 214 \mathrm{~nm}$

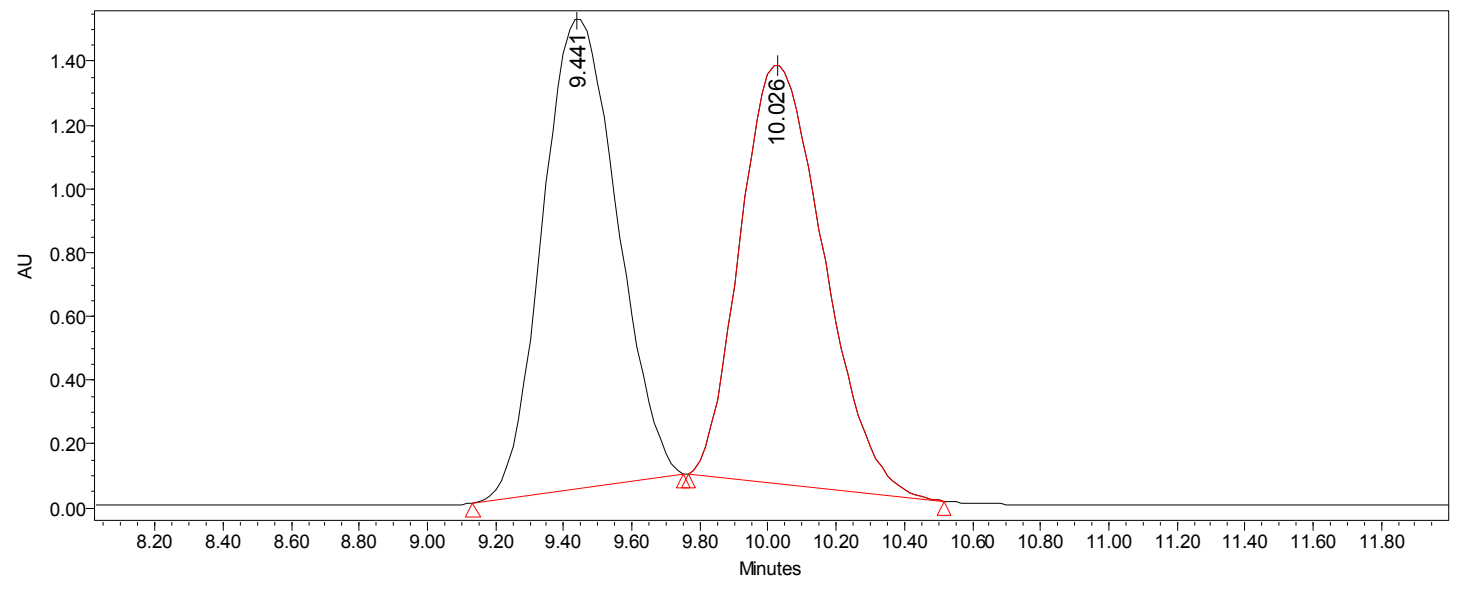

\begin{tabular}{|r|r|l|r|}
\hline & Retention Time & Area & \% Area \\
\hline 1 & 9.441 & 22497132 & 50.18 \\
\hline 2 & 10.026 & 22332979 & 49.82 \\
\hline
\end{tabular}
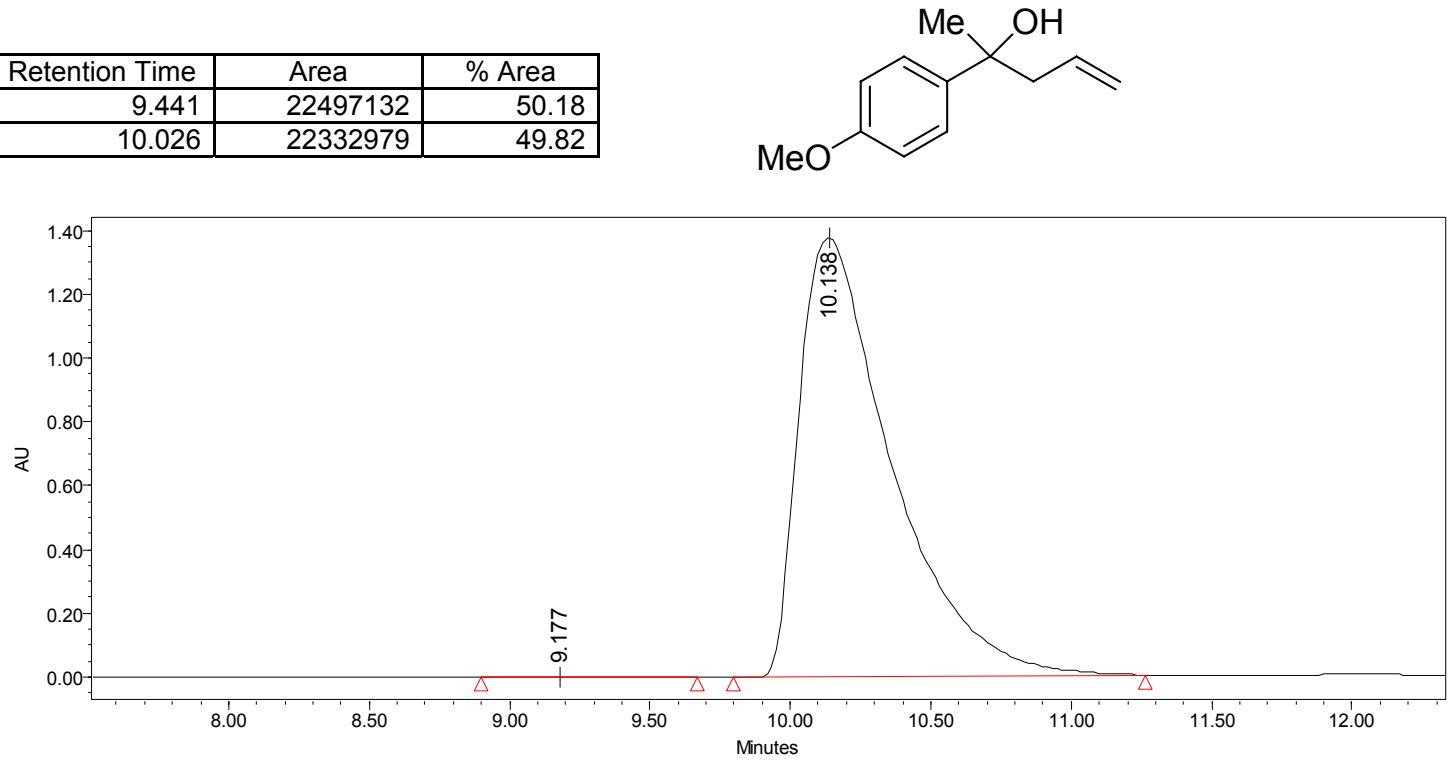

\begin{tabular}{|r|r|r|r|}
\hline & Retention Time & Area & $\%$ Area \\
\hline 1 & 9.177 & 31281 & 0.10 \\
\hline 2 & 10.138 & 31455156 & 99.90 \\
\hline
\end{tabular}<smiles>C=CC[C@](C)(O)c1ccc(OC)cc1</smiles> 
Table 2, entry 6: 11f Chiralpak ${ }^{\circledR}$ AD-H Column, Hexane:IPA = 99:1, $1.0 \mathrm{~mL} / \mathrm{min}, 214 \mathrm{~nm}$

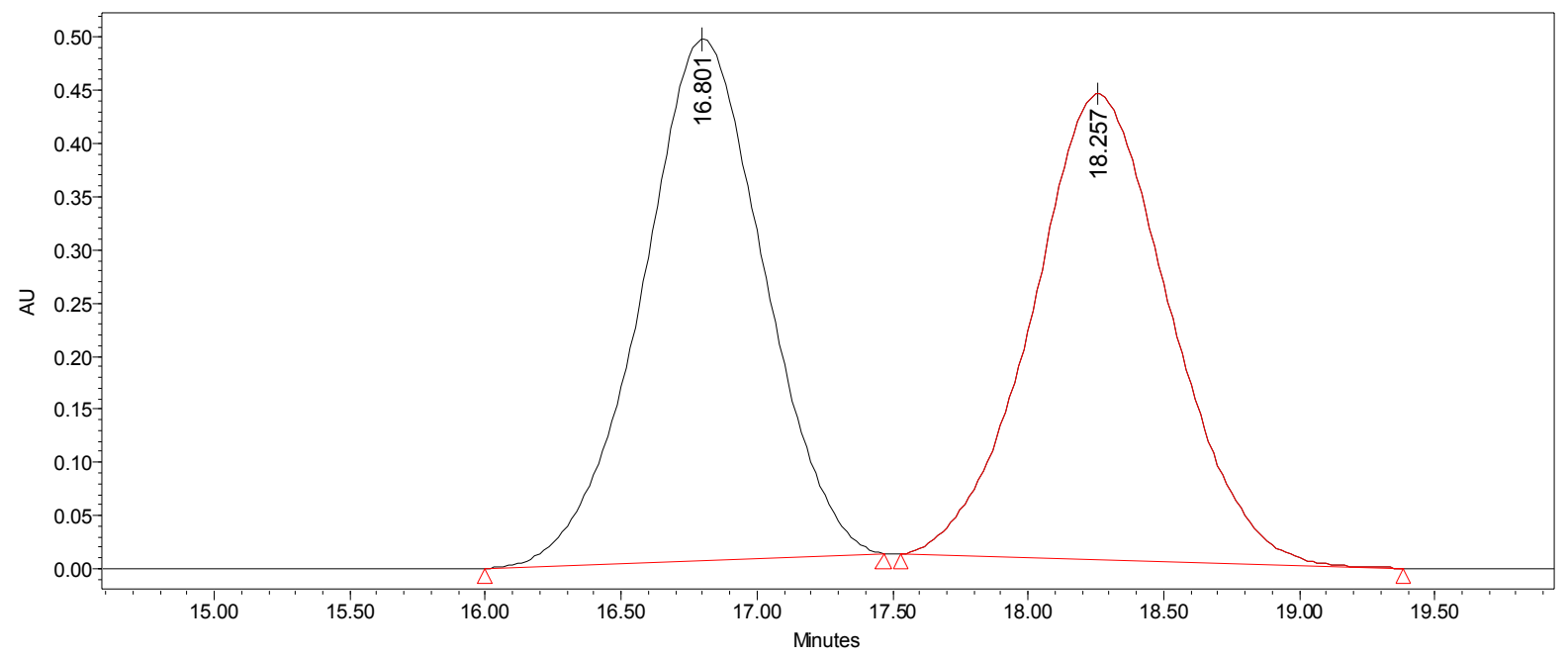

\begin{tabular}{|l|r|r|r|}
\hline & Retention Time & Area & \% Area \\
\hline 1 & 16.772 & 118659504 & 48.31 \\
\hline 2 & 18.272 & 126978155 & 51.69 \\
\hline
\end{tabular}

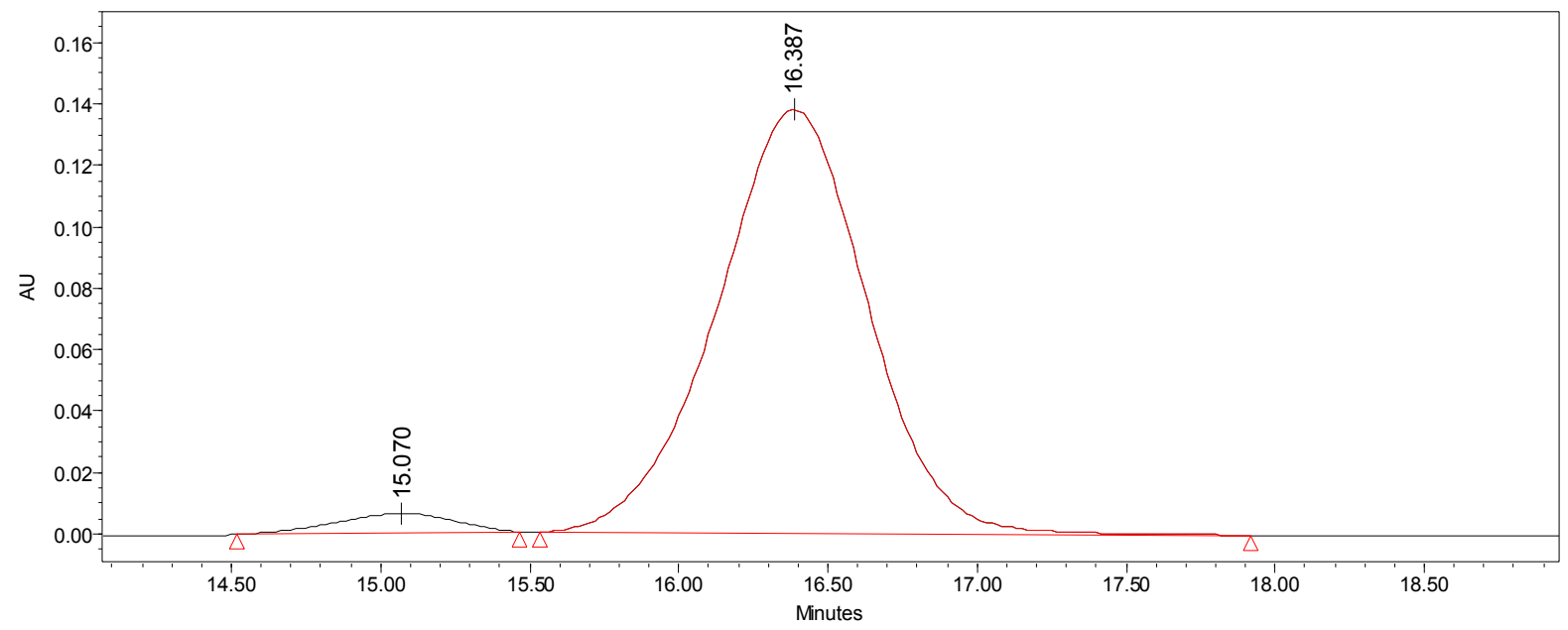

\begin{tabular}{|l|r|r|r|}
\hline & Retention Time & Area & $\%$ Area \\
\hline 1 & 15.070 & 177464 & 3.54 \\
\hline 2 & 16.387 & 4838350 & 96.46 \\
\hline
\end{tabular}<smiles>C=CC[C@@](C)(O)c1ccc2ccccc2c1</smiles> 
Table 2, entry 7: 11g Chiralpak ${ }^{\circledR}$ AD-H Column, Hexane:IPA = 999:1, $1.0 \mathrm{~mL} / \mathrm{min}, 230 \mathrm{~nm}$

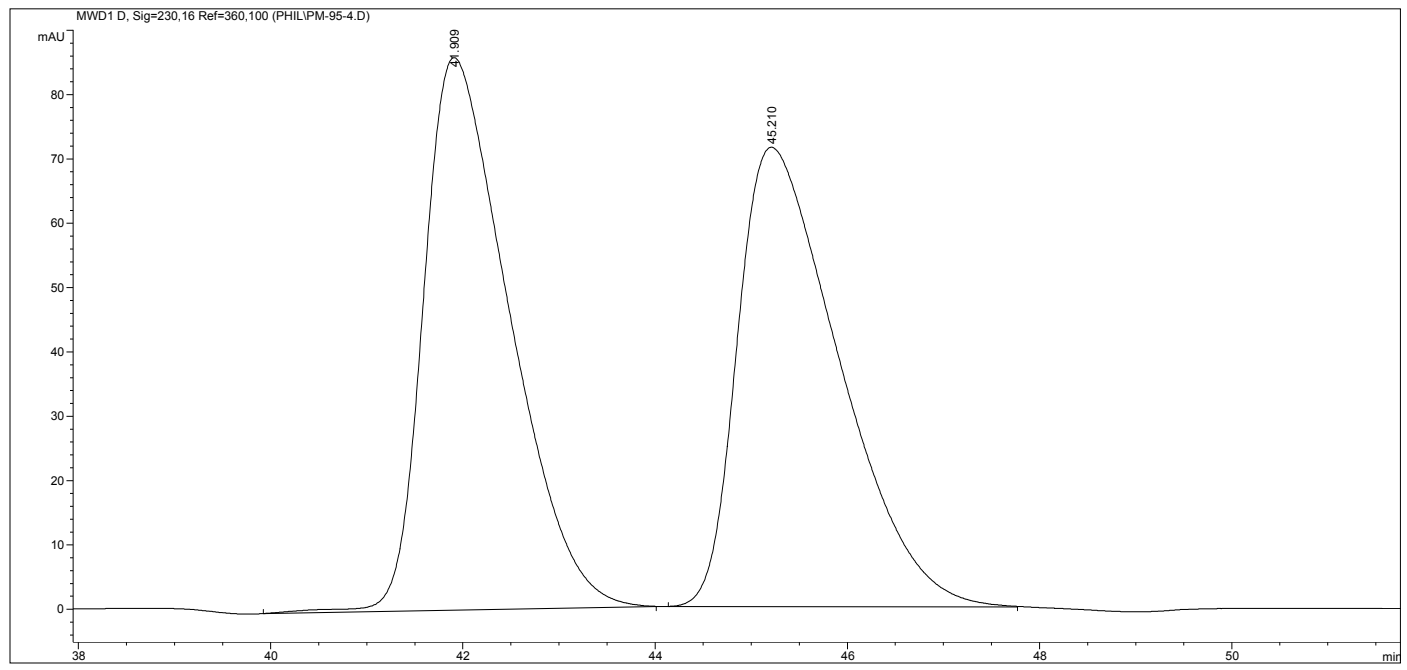

Signal 4: MWD1 D, Sig=230,16 Ref $=360,100$
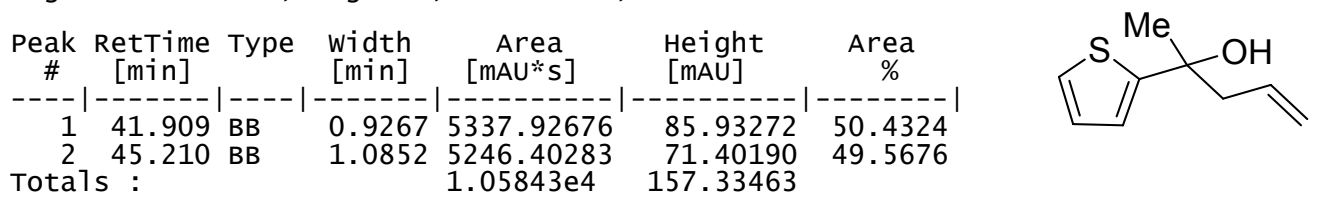
Totals :

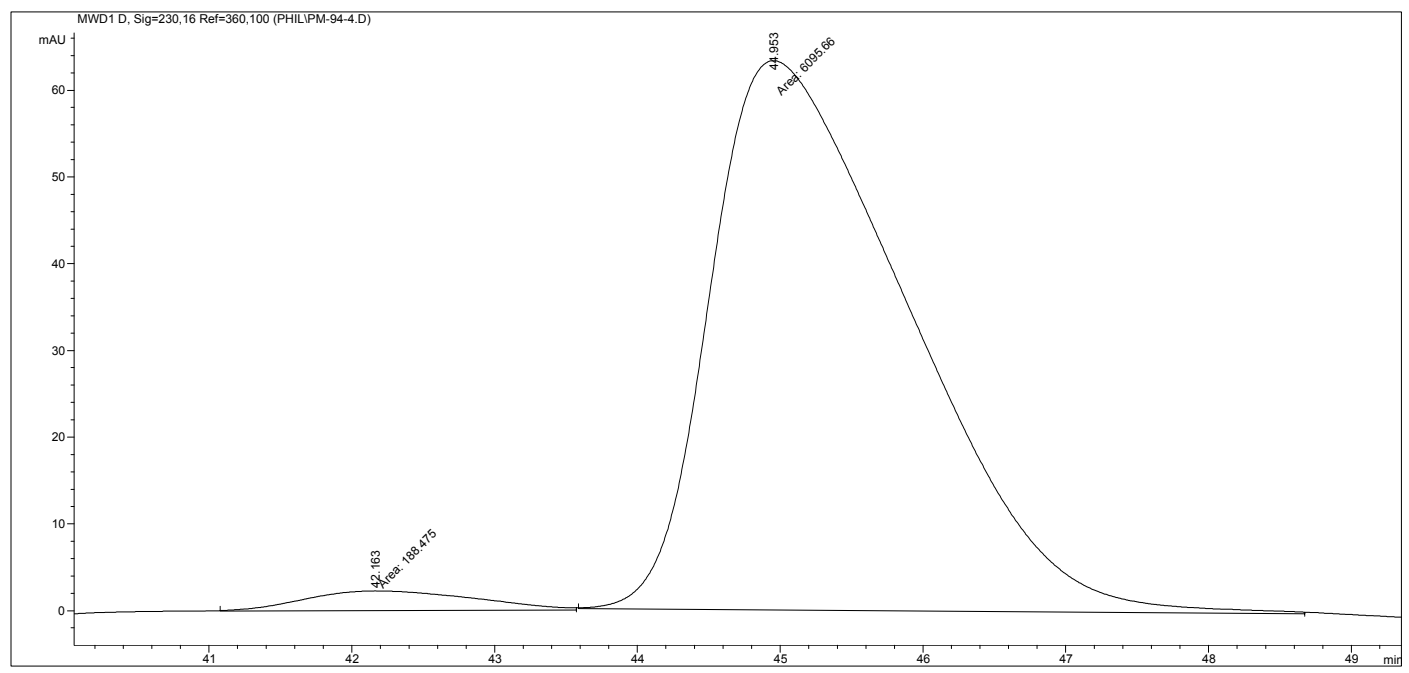

Signal 4: MWD1 D, Sig=230,16 Ref=360,100
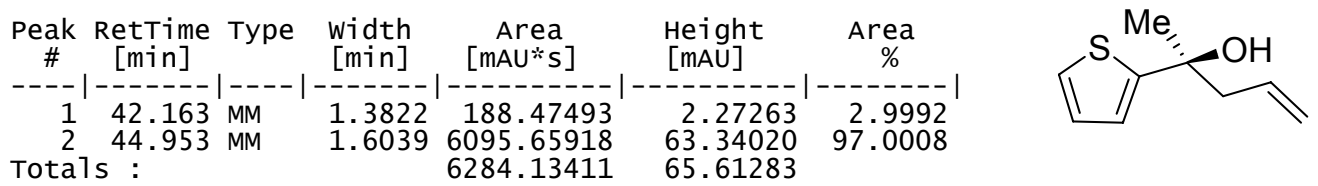

Totals : 
Table 2, entry 8: 11h Chiralpak ${ }^{\circledR}$ AD-H Column, Hexane:IPA = 999:1, $1.0 \mathrm{~mL} / \mathrm{min}, 230 \mathrm{~nm}$

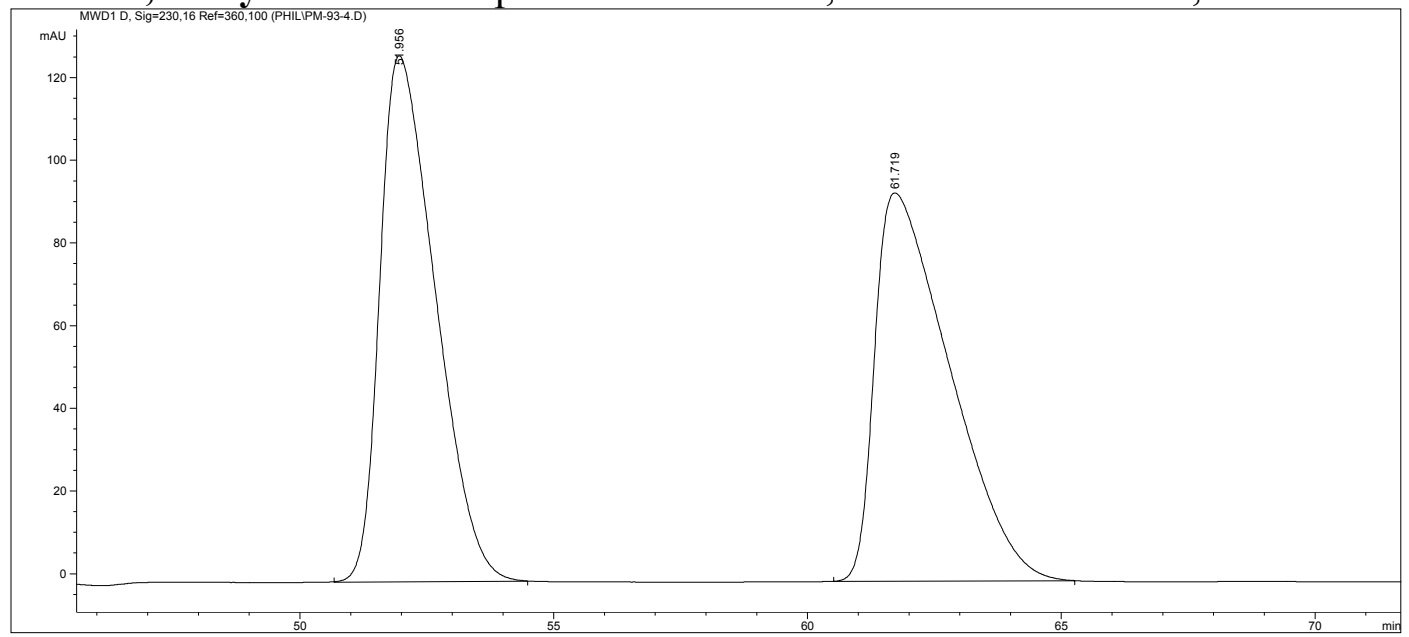

Signal 4: MWD1 D, Sig=230,16 Ref $=360,100$

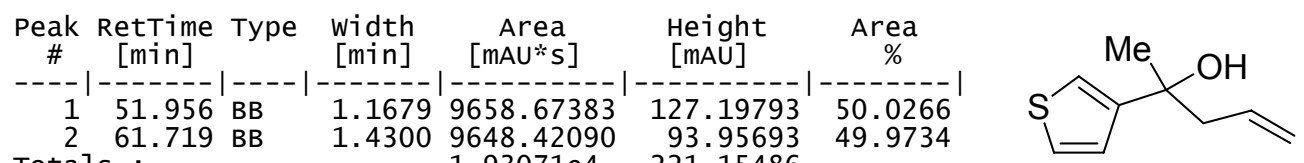
Totals

$9648.42090 \quad 93.95693$

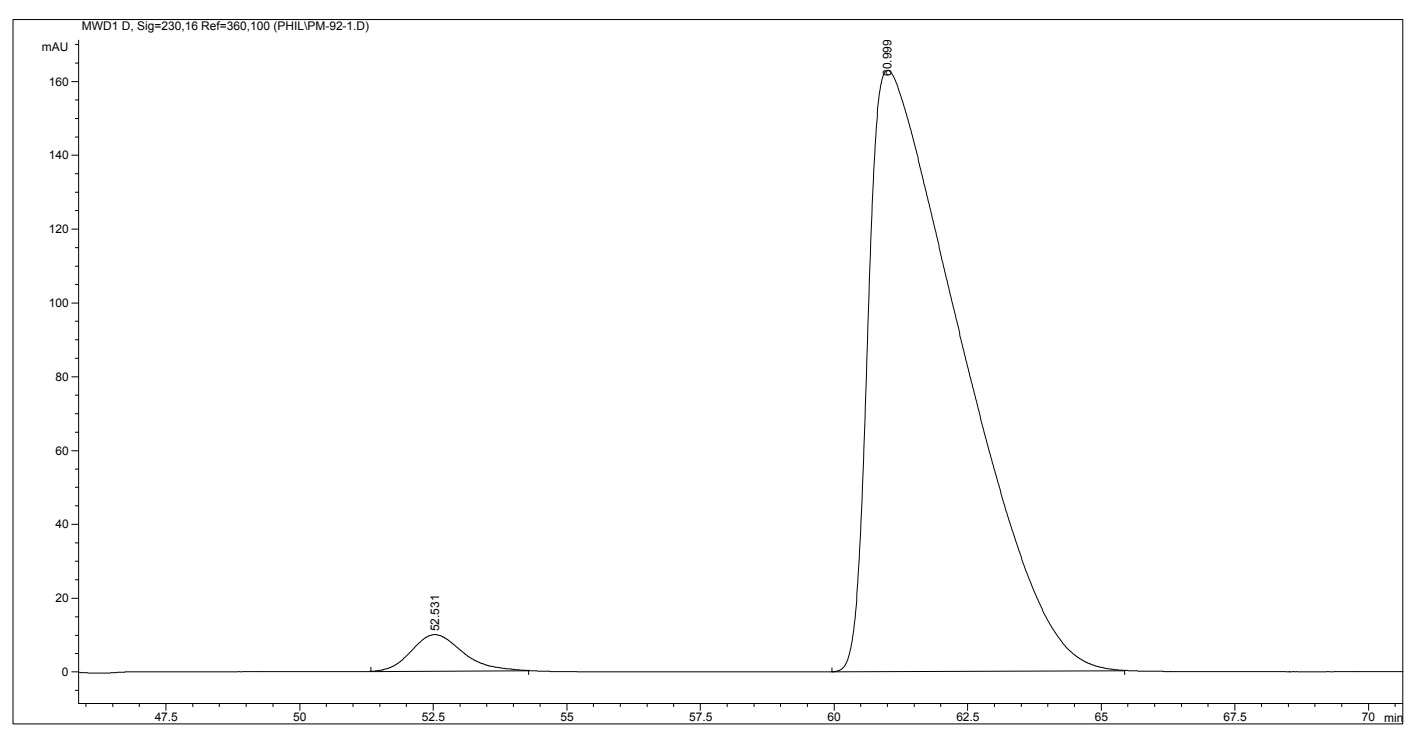

Signal 4: MWD1 D, Sig=230,16 Ref=360,100
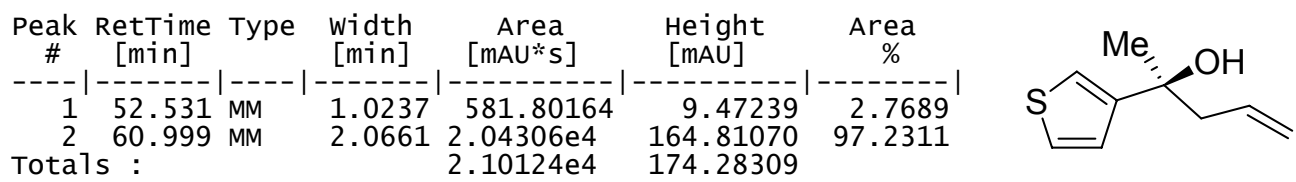
Totals : 
Table 2, entry 9: 11i Chiralpak ${ }^{\circledR}$ AD-H Column, Hexane:IPA = 99:1, $1.0 \mathrm{~mL} / \mathrm{min}, 214 \mathrm{~nm}$

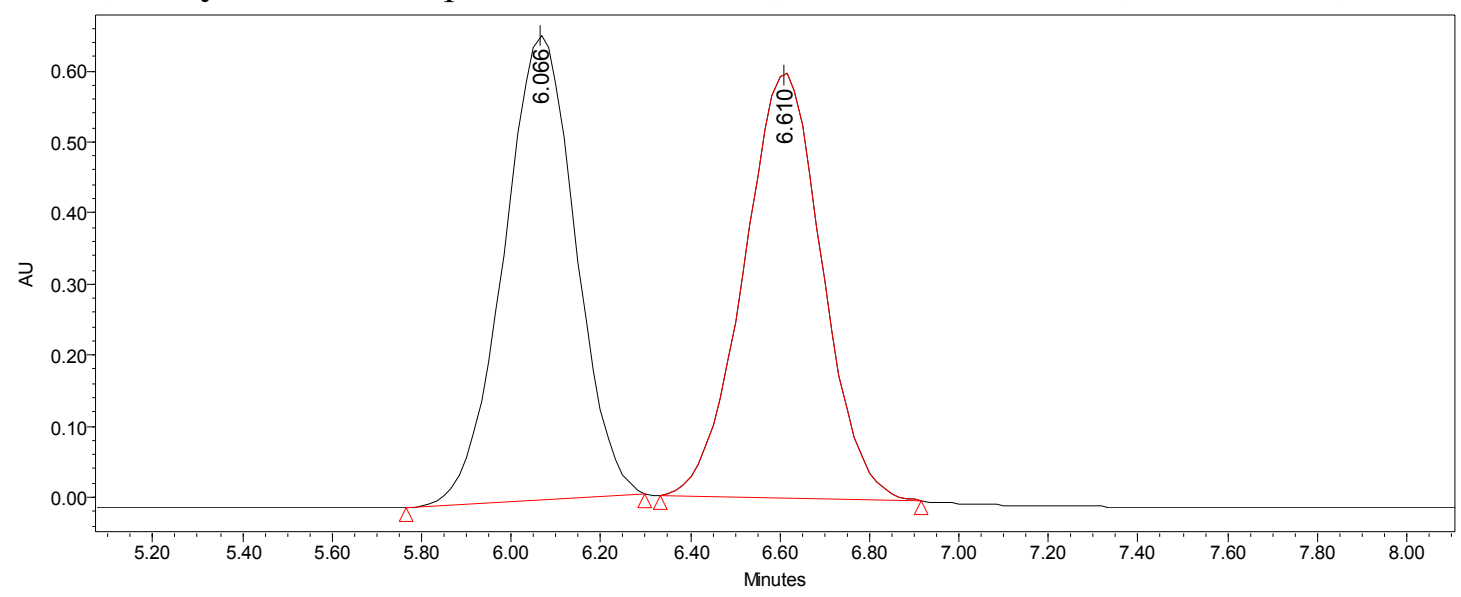

\begin{tabular}{|r|r|r|r|}
\hline & Retention Time & Area & $\%$ Area \\
\hline 1 & 6.066 & 7275498 & 49.85 \\
\hline 2 & 6.610 & 7320204 & 50.15 \\
\hline
\end{tabular}

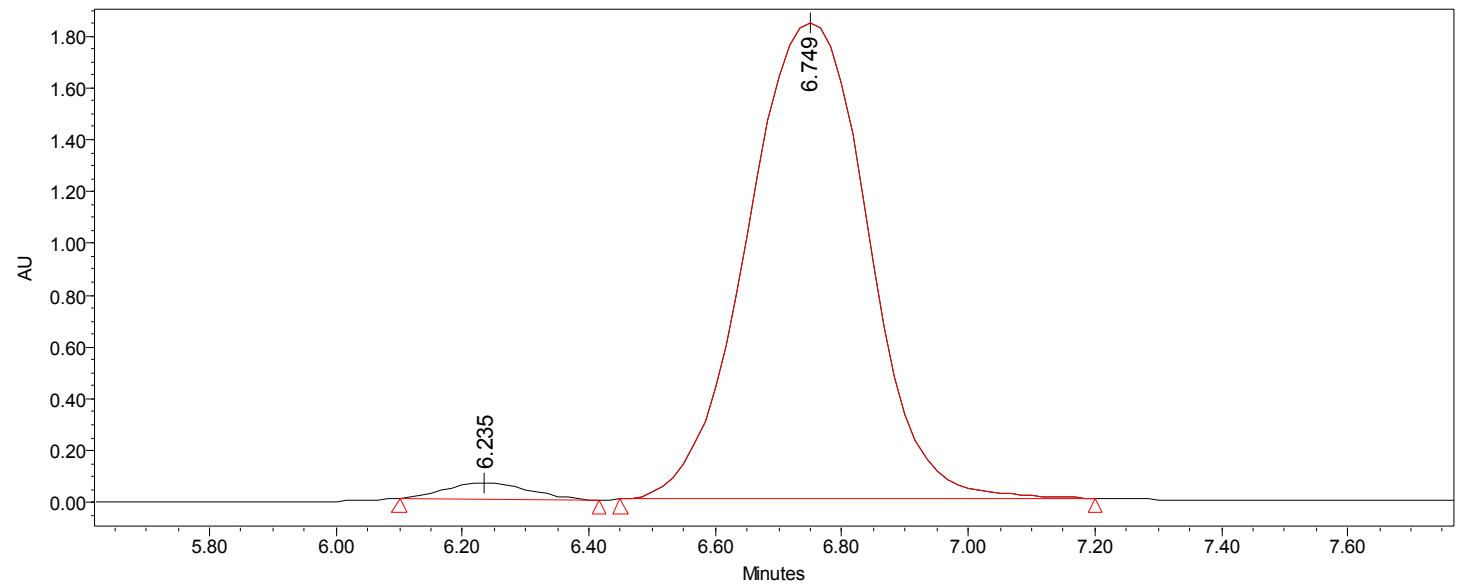

\begin{tabular}{|r|r|r|r|}
\hline & Retention Time & Area & \% Area \\
\hline 1 & 6.235 & 619379 & 2.46 \\
\hline 2 & 6.749 & 24537200 & 97.54 \\
\hline
\end{tabular}<smiles>C=CCC(O)(CC)c1ccccc1</smiles> 
Table 2, entry 10: 11j Chiralpak ${ }^{\circledR}$ AD-H Column, Hexane:IPA = 99:1, $1.0 \mathrm{~mL} / \mathrm{min}, 214 \mathrm{~nm}$

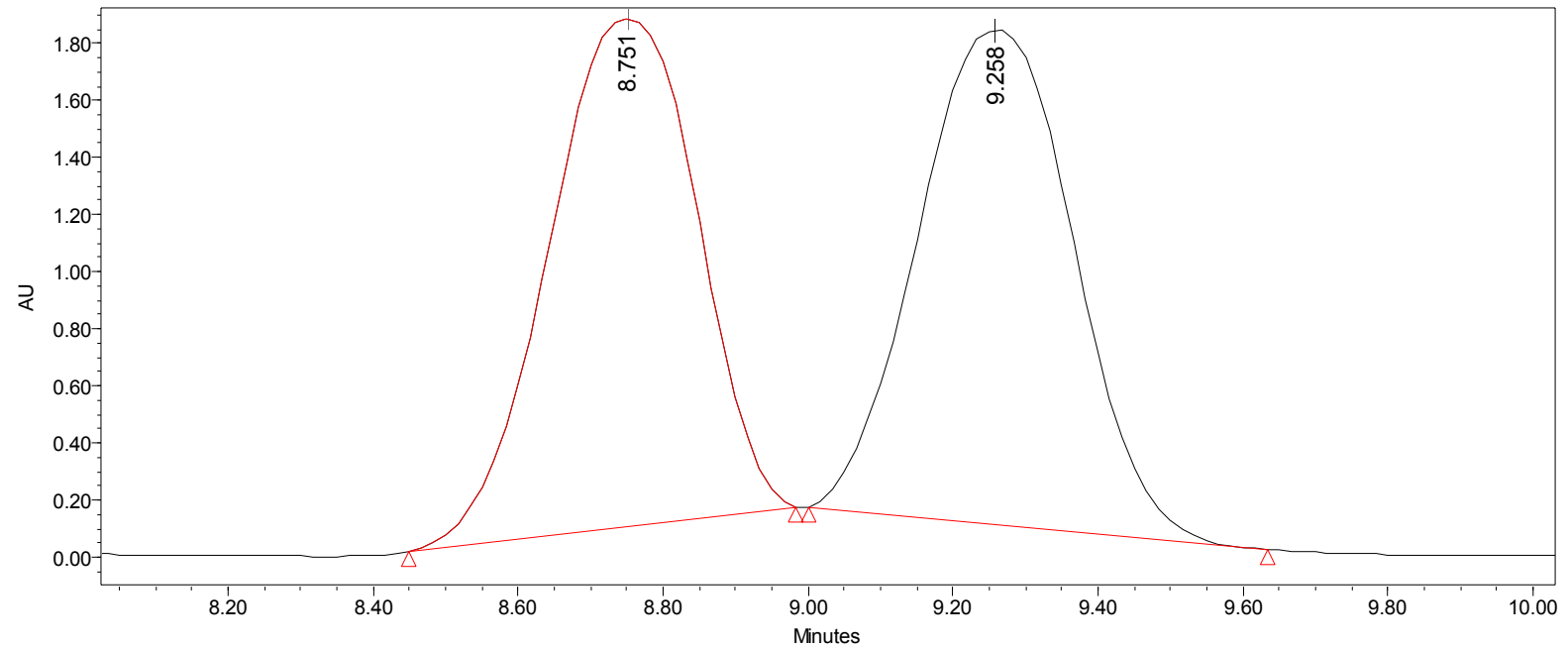

\begin{tabular}{|l|r|l|r|}
\hline & Retention Time & Area & \% Area \\
\hline 1 & 8.751 & 25265681 & 49.41 \\
\hline 2 & 9.258 & 25870163 & 50.59 \\
\hline
\end{tabular}
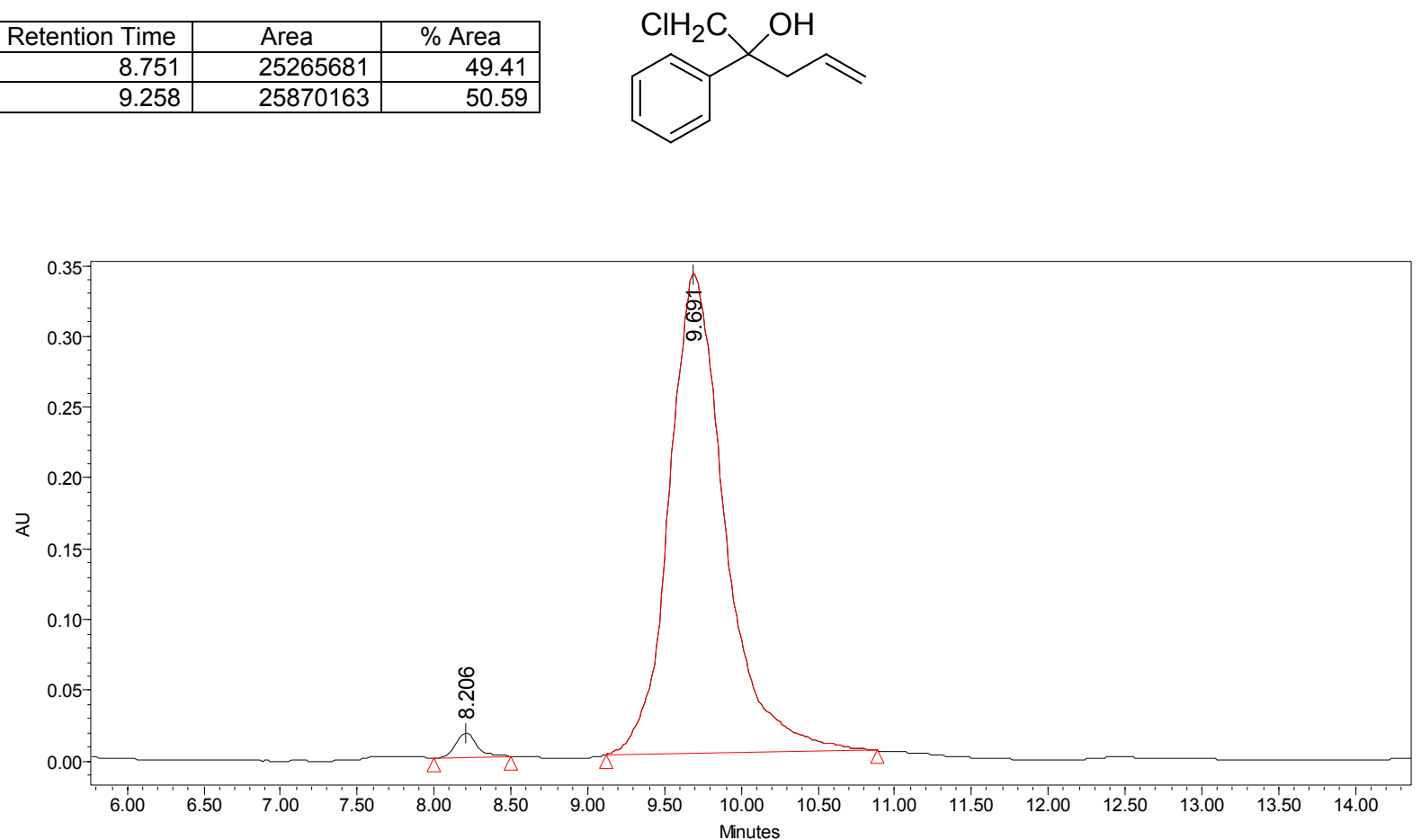

\begin{tabular}{|r|r|r|r|}
\hline & Retention Time & Area & \% Area \\
\hline 1 & 8.206 & 159526 & 1.83 \\
\hline 2 & 9.691 & 8564743 & 98.17 \\
\hline
\end{tabular}

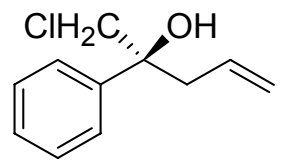


Table 2, entry 11: 11k Chiralcel ${ }^{\circledR}$ OD-H Column, Hexane:IPA $=98: 2,1.0 \mathrm{~mL} / \mathrm{min}, 214 \mathrm{~nm}$

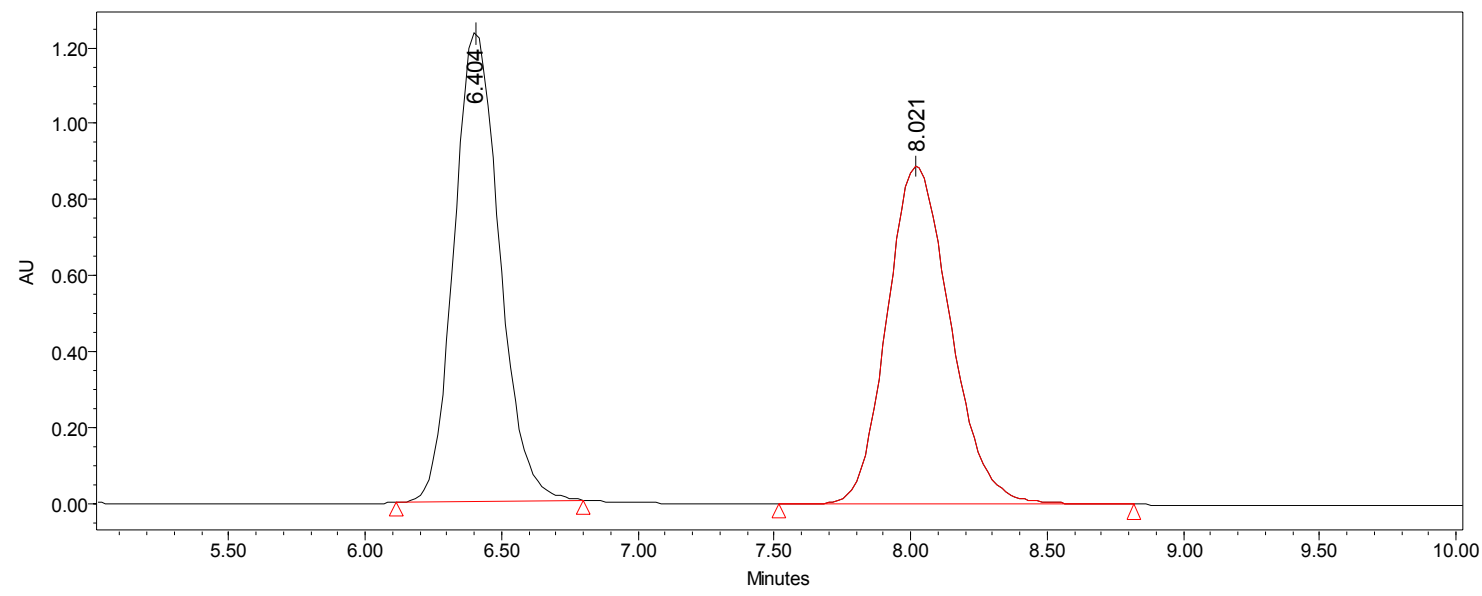

\begin{tabular}{|l|r|r|r|}
\hline & Retention Time & Area & $\%$ Area \\
\hline 1 & 6.404 & 14341456 & 49.79 \\
\hline 2 & 8.021 & 14461913 & 50.21 \\
\hline
\end{tabular}
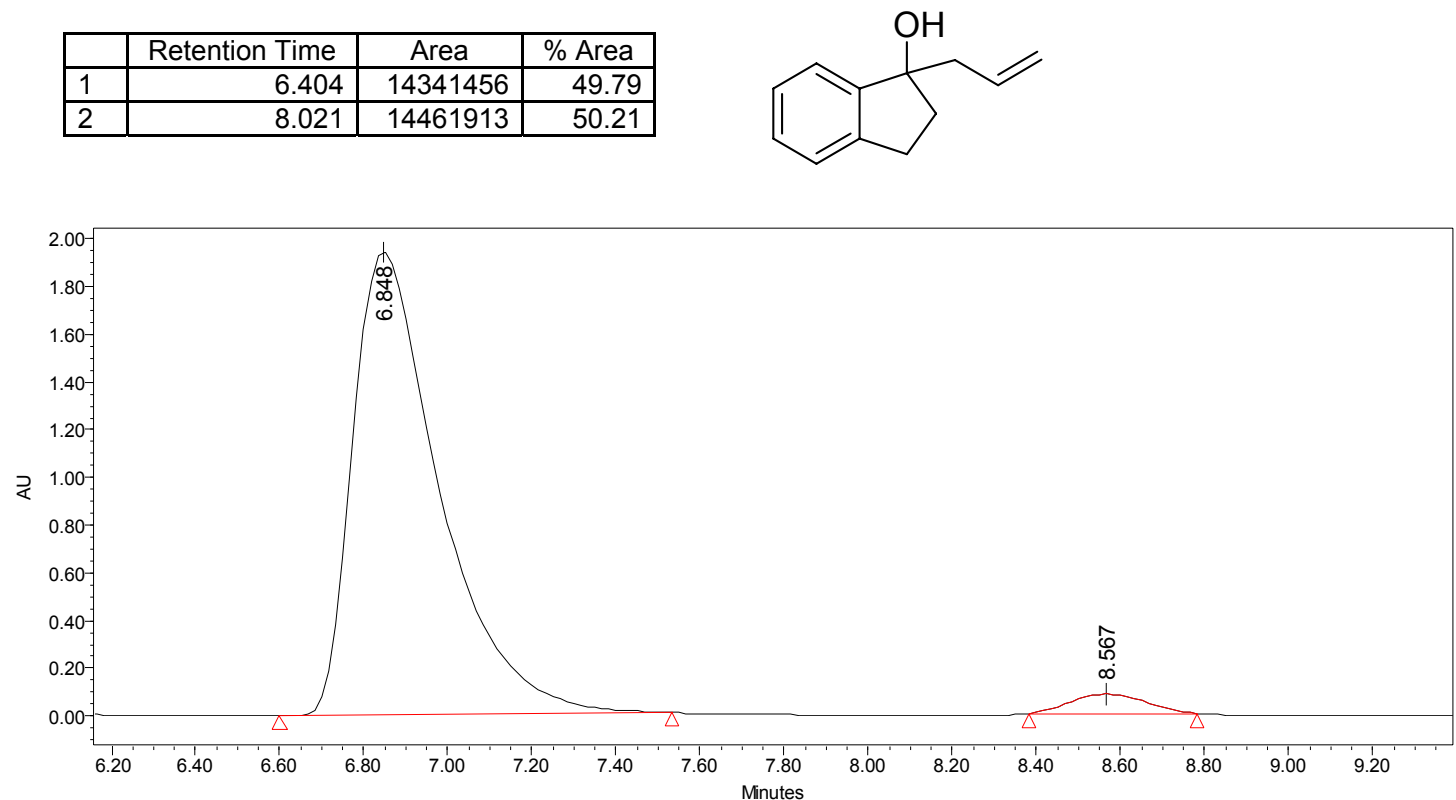

\begin{tabular}{|r|r|r|r|}
\hline & Retention Time & Area & \% Area \\
\hline 1 & 6.848 & 27703502 & 96.40 \\
\hline 2 & 8.567 & 1035546 & 3.60 \\
\hline
\end{tabular}<smiles>C=CCC1(O)CCc2ccccc21</smiles> 
Table 2, entry 12: 11 Chiralcel ${ }^{\circledR}$ OD-H Column, Hexane:IPA $=98: 2,1.0 \mathrm{~mL} / \mathrm{min}, 214 \mathrm{~nm}$

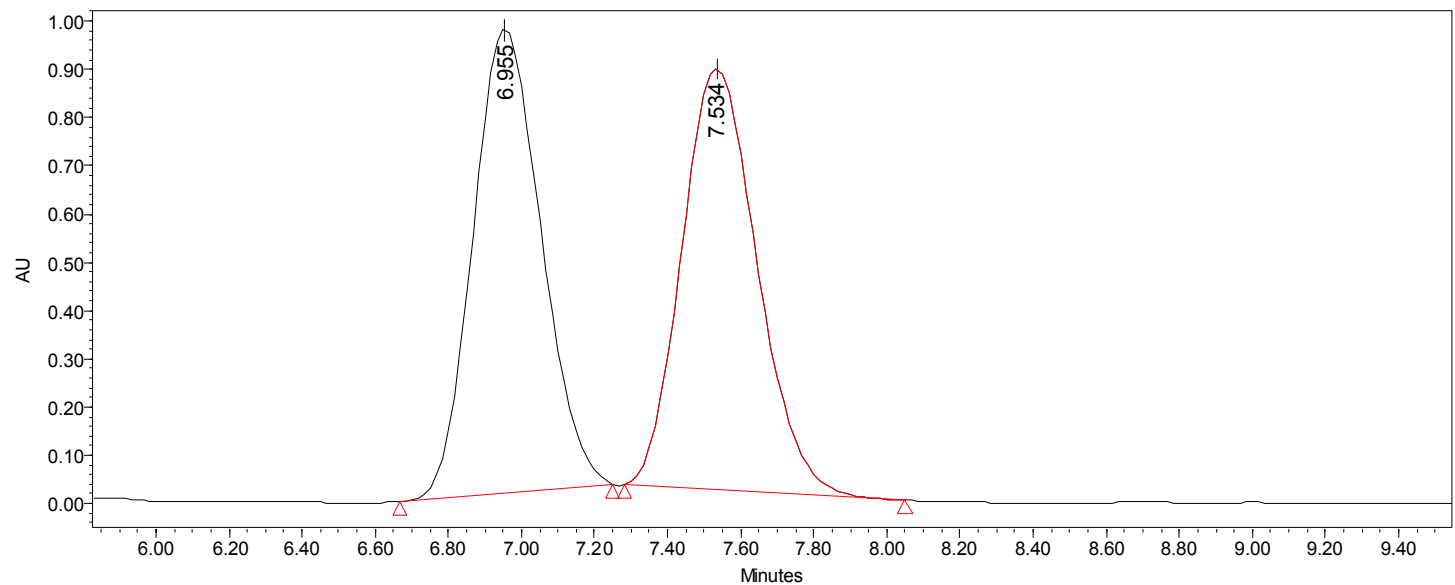

\begin{tabular}{|r|r|r|r|}
\hline & Retention Time & Area & \% Area \\
\hline 1 & 6.955 & 12484847 & 50.32 \\
\hline 2 & 7.534 & 12326624 & 49.68 \\
\hline
\end{tabular}
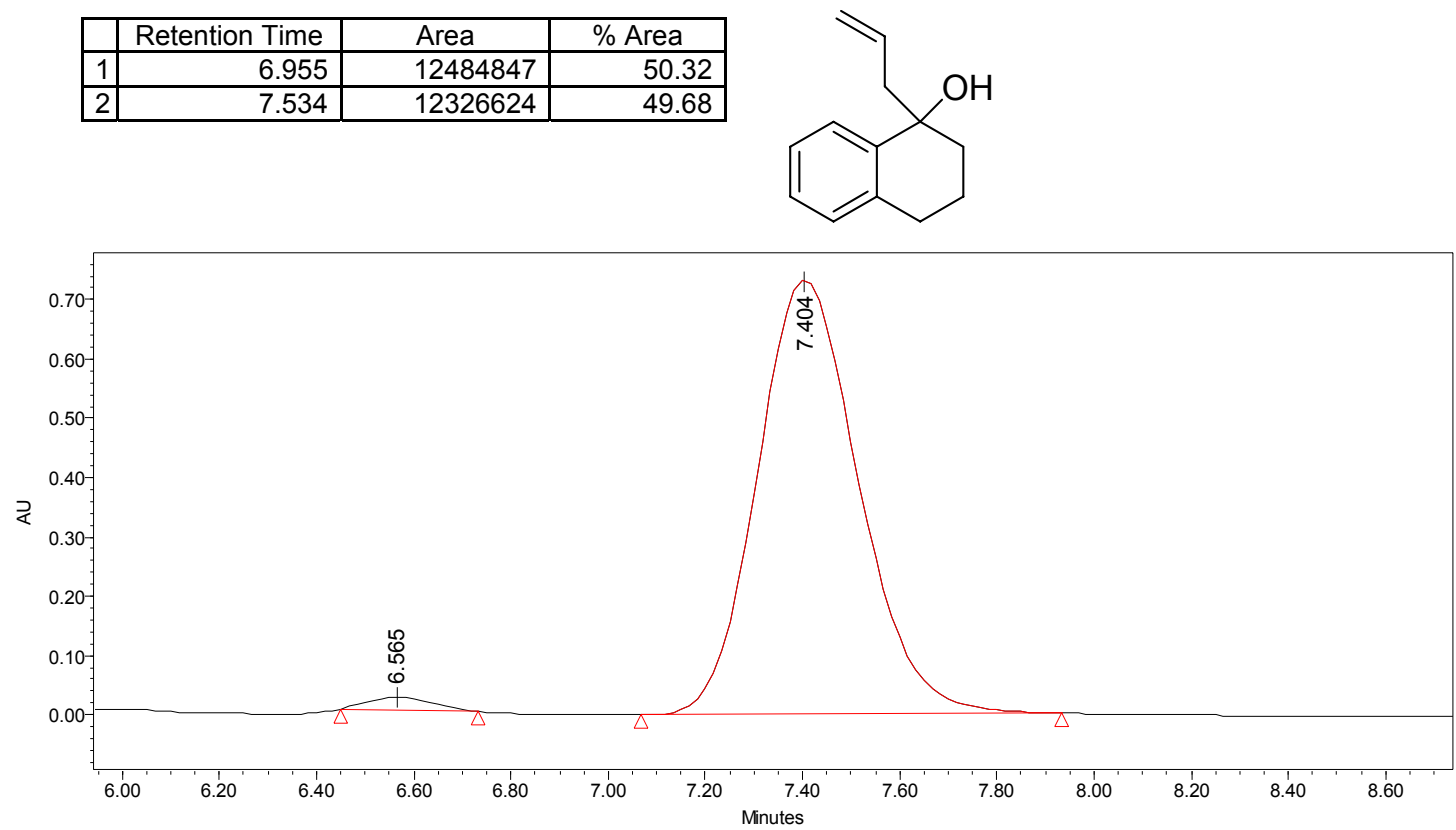

\begin{tabular}{|r|r|r|r|}
\hline & Retention Time & Area & \% Area \\
\hline 1 & 6.565 & 257219 & 2.37 \\
\hline 2 & 7.404 & 10608317 & 97.63 \\
\hline
\end{tabular}<smiles>C=CC[C@]1(O)CCCc2ccccc21</smiles> 
Table 2, entry 13: 11m Chiralcel ${ }^{\circledR}$ OD-H Column, Hexane:IPA $=98: 2,1.0 \mathrm{~mL} / \mathrm{min}, 214 \mathrm{~nm}$

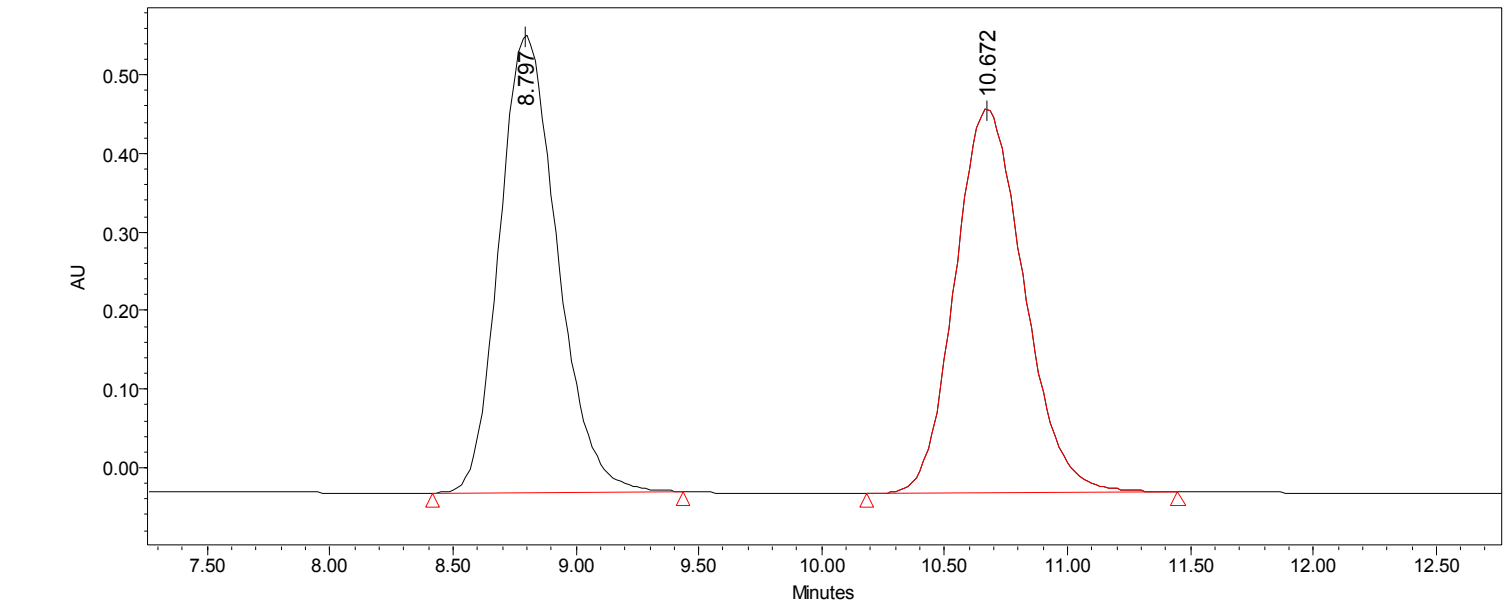

\begin{tabular}{|r|r|r|r|}
\hline & Retention Time & Area & \% Area \\
\hline 1 & 8.797 & 9527777 & 50.08 \\
\hline 2 & 10.672 & 9495838 & 49.92 \\
\hline
\end{tabular}<smiles>C=CCC1(O)CCOc2ccccc21</smiles>

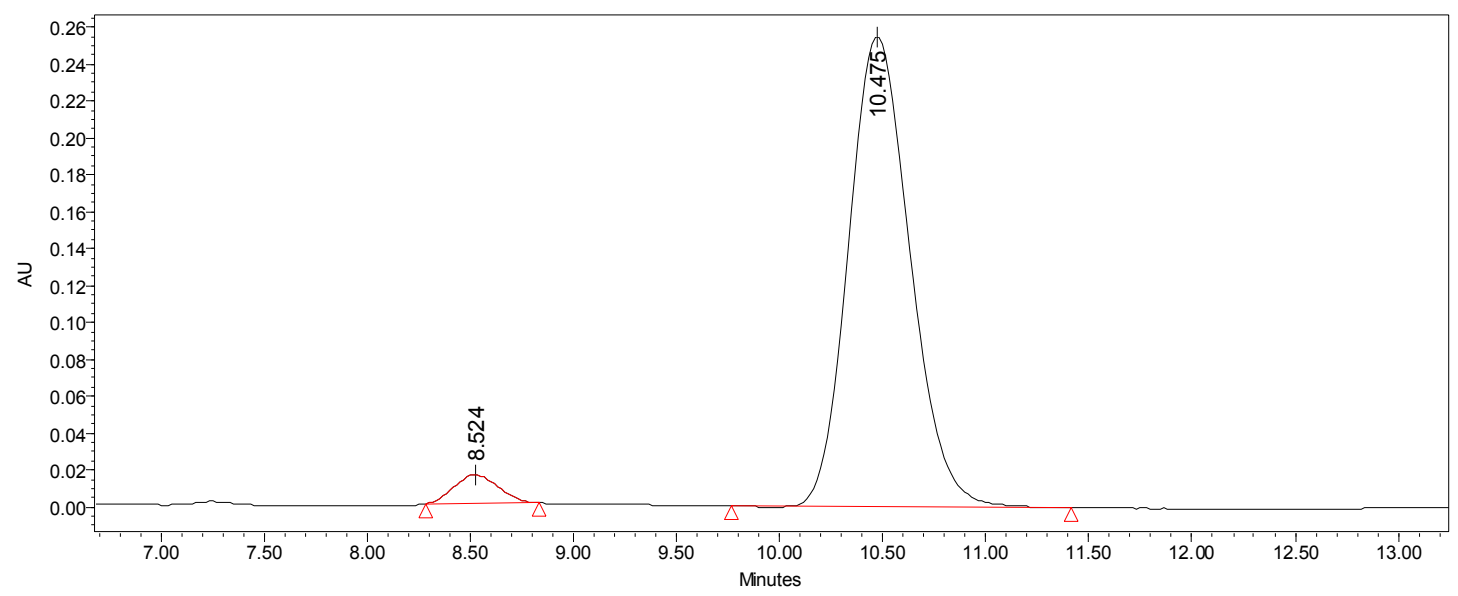

\begin{tabular}{|r|r|r|r|}
\hline & Retention Time & Area & \% Area \\
\hline 1 & 8.524 & 207217 & 3.77 \\
\hline 2 & 10.475 & 5295730 & 96.23 \\
\hline
\end{tabular}<smiles>C=CC[C@]1(O)CCOc2ccccc21</smiles> 
Table 2, entry 14: 11n ChiralGC, Chiraldex B-DM column, $30 \mathrm{~m} \times 0.25 \mathrm{~mm}$ I.D., $60-170^{\circ} \mathrm{C}$, 30min, FID
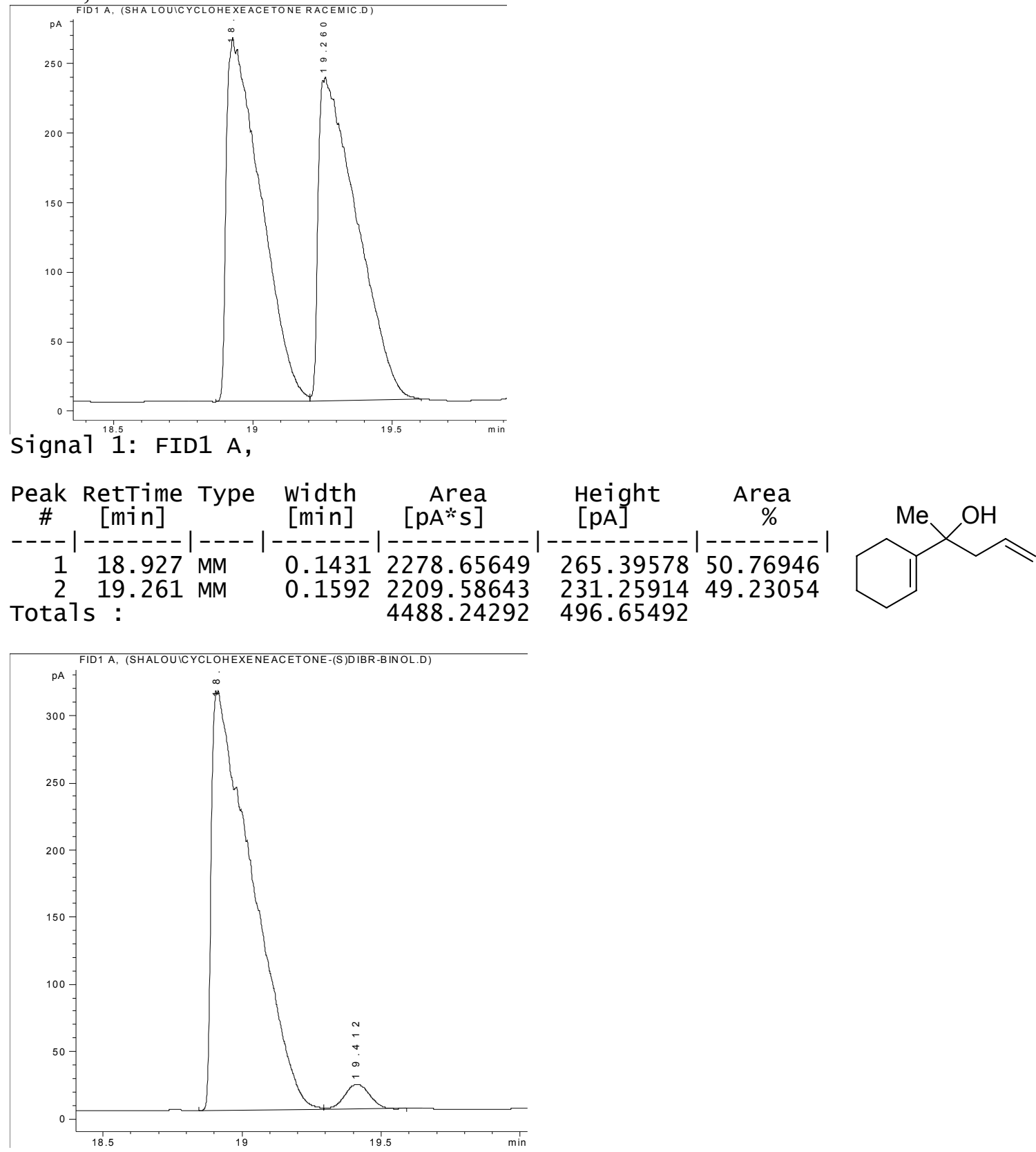

Signa1 1: FID1 A,

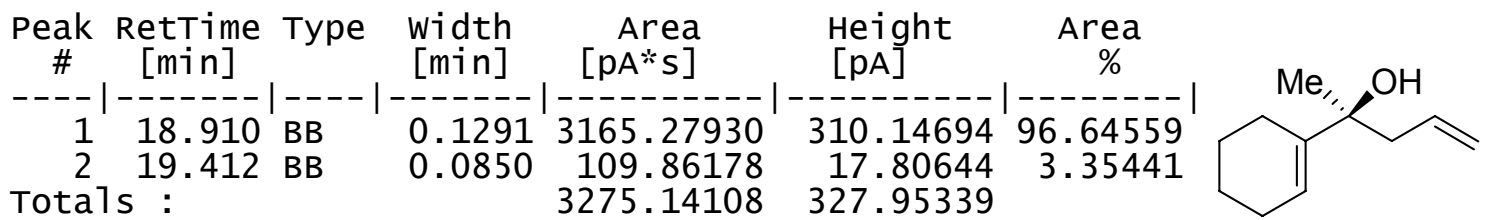


Table 2, entry 15: 110 Chiralcel $^{\circledR}$ OD-H Column, Hexane:IPA $=95: 5,1.0 \mathrm{~mL} / \mathrm{min}, 214 \mathrm{~nm}$
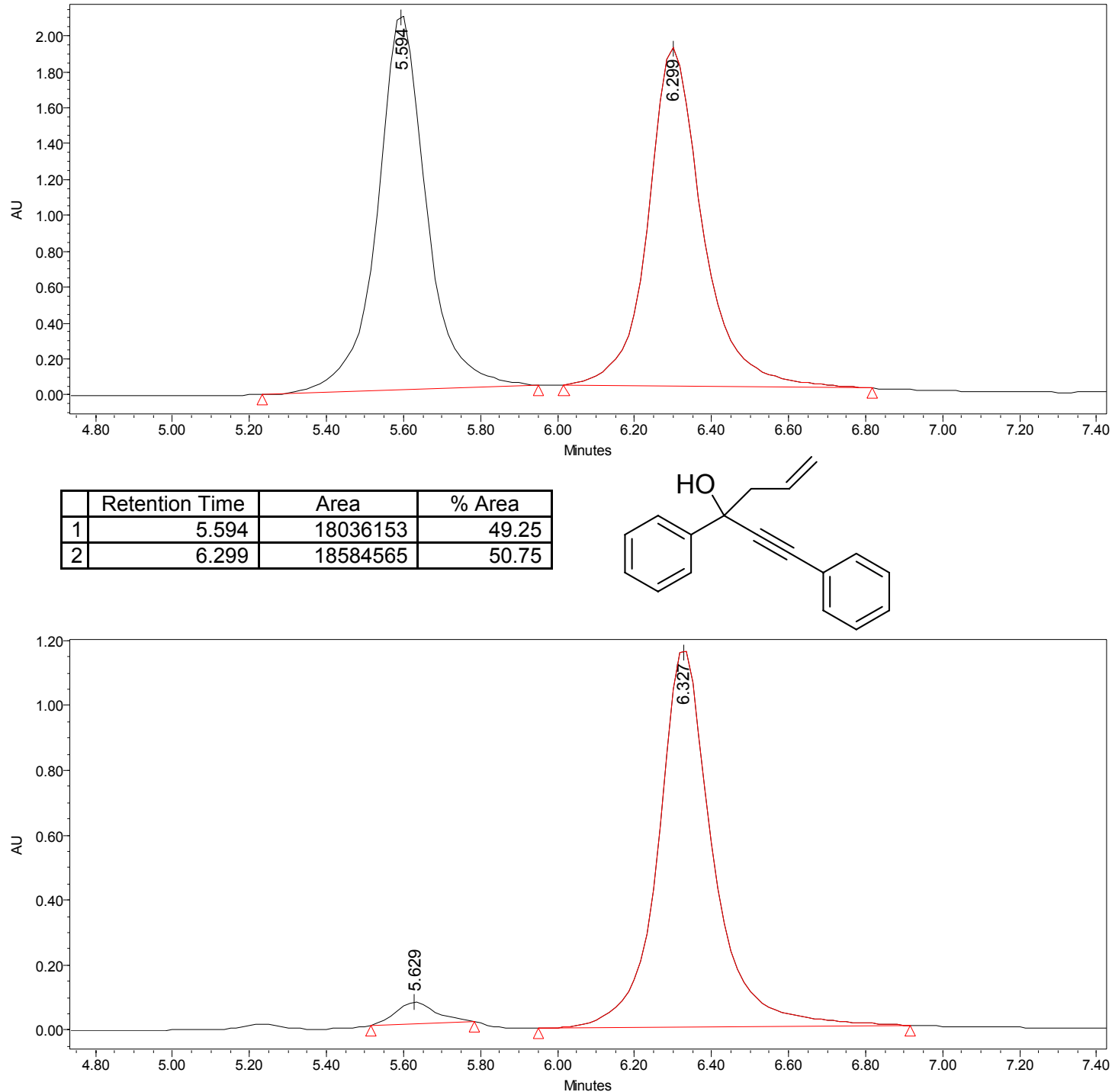

\begin{tabular}{|r|r|r|r|}
\hline & Retention Time & Area & \% Area \\
\hline 1 & 5.629 & 610630 & 4.94 \\
\hline 2 & 6.327 & 11745517 & 95.06 \\
\hline
\end{tabular}

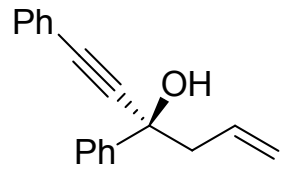


Scheme 1: Chiralpak ${ }^{\circledR}$ AD-H Column, Hexane:IPA = 999:1, $1.0 \mathrm{~mL} / \mathrm{min}, 214 \mathrm{~nm}$

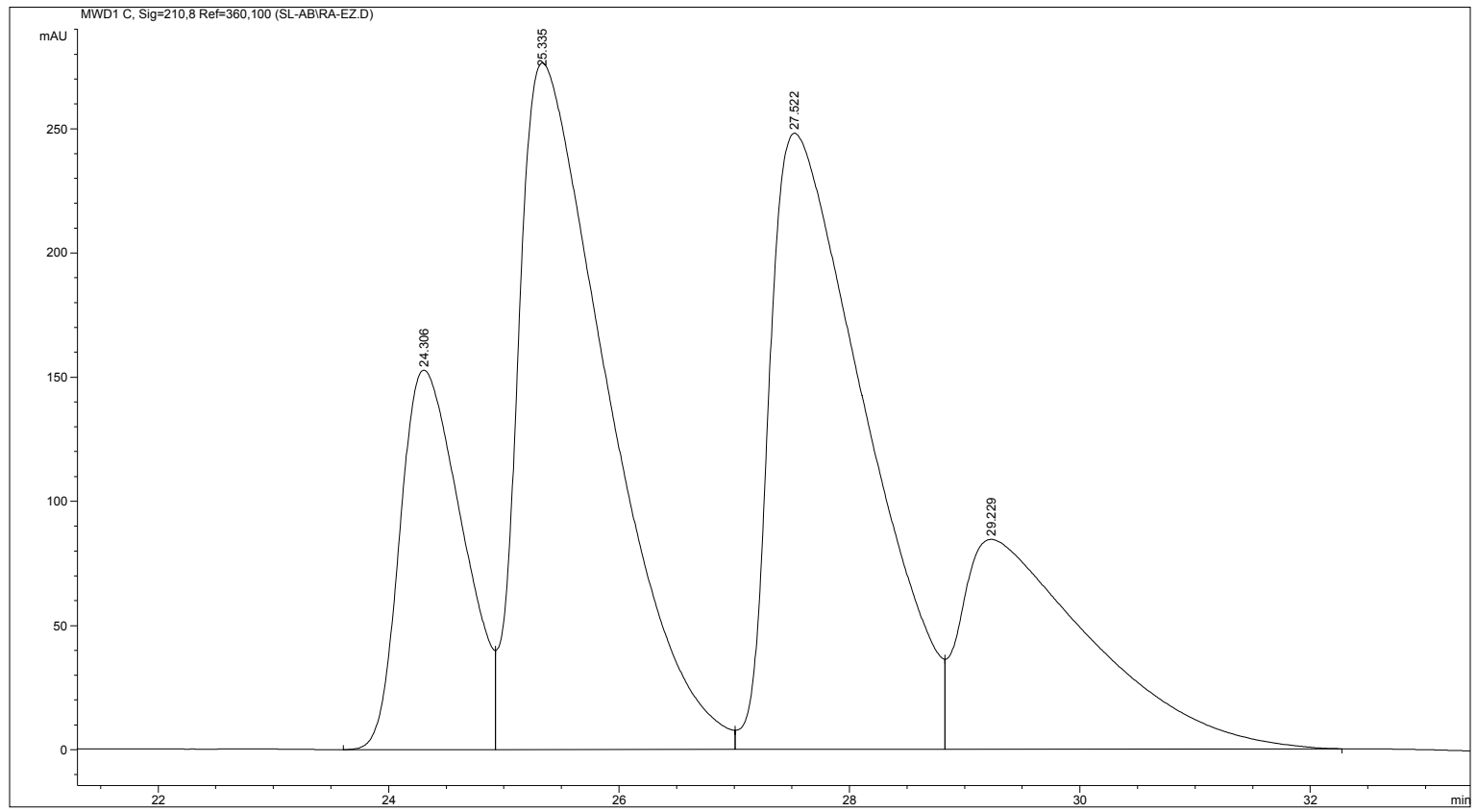

Signal 3: MWD1 C, Sig=210,8 Ref $=360,100$

Peak RetTime Type width Area Height Area

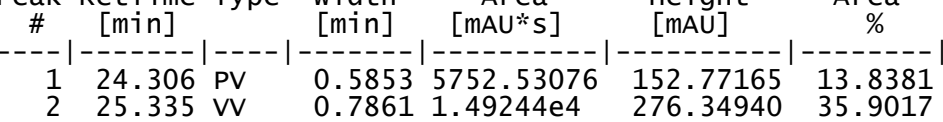

$\begin{array}{llllll}2 & 25.335 \mathrm{VV} & 0.7861 & 1.49244 \mathrm{e} 4 & 276.34940 & 35.9017 \\ 3 & 27.522 \mathrm{VV} & 0.8384 & 1.42130 \mathrm{e} 4 & 248.06790 & 34.1902\end{array}$

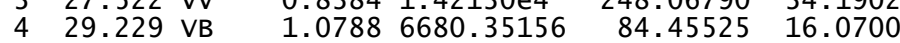

Totals:

$4.15703 \mathrm{e} 4 \quad 761.64420$

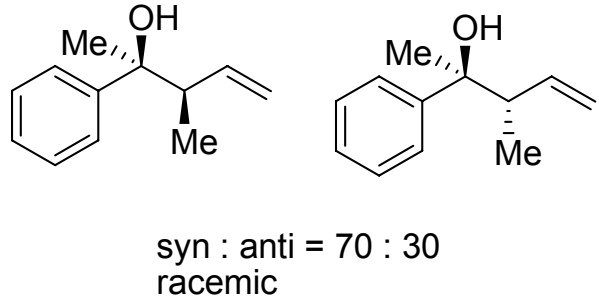


anti-13a: Chiralpak ${ }^{\circledR}$ AD-H Column, Hexane:IPA = 999:1, $1.0 \mathrm{~mL} / \mathrm{min}, 210 \mathrm{~nm}$

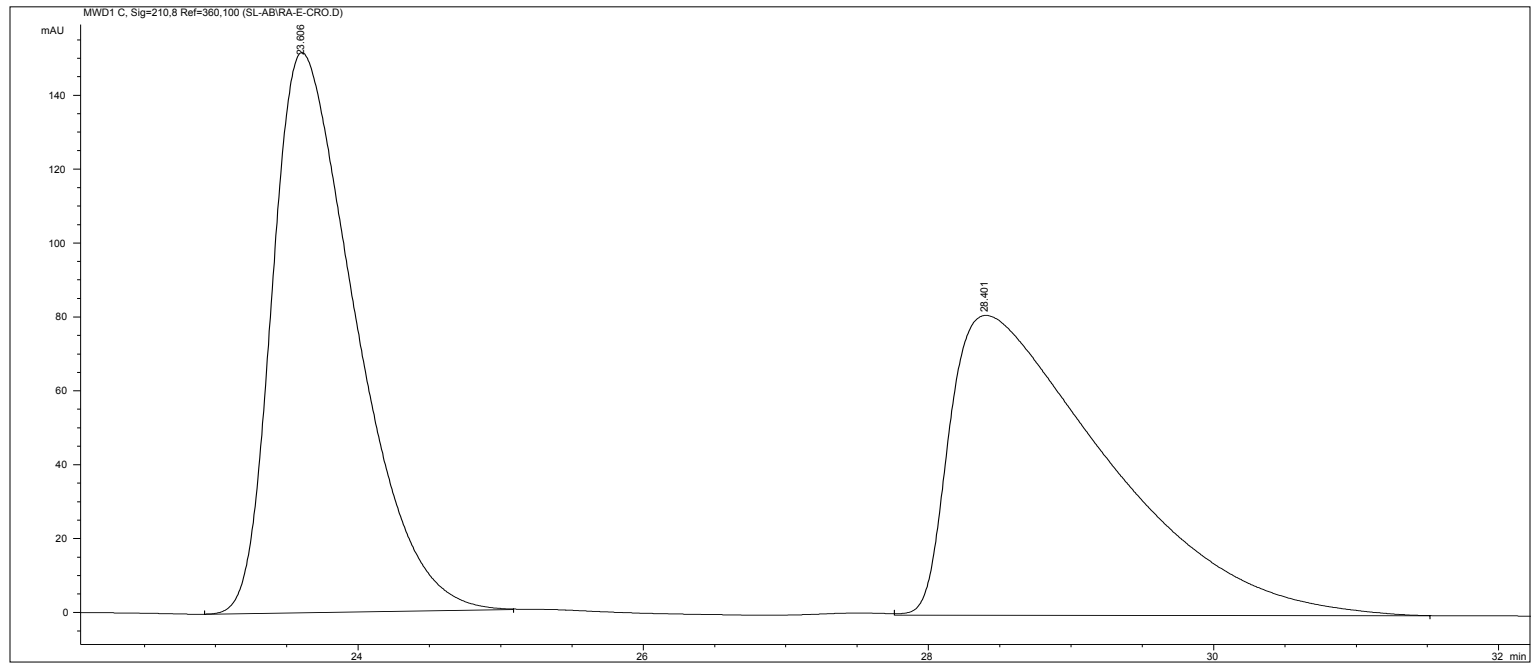

Signa1 3: MWD1 C, Sig $=210,8$ Ref $=360,100$

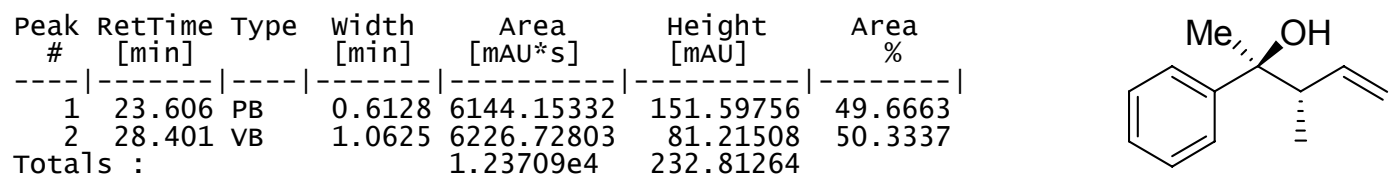

Totals :

$1.23709 \mathrm{e} 4 \quad 232.81264$

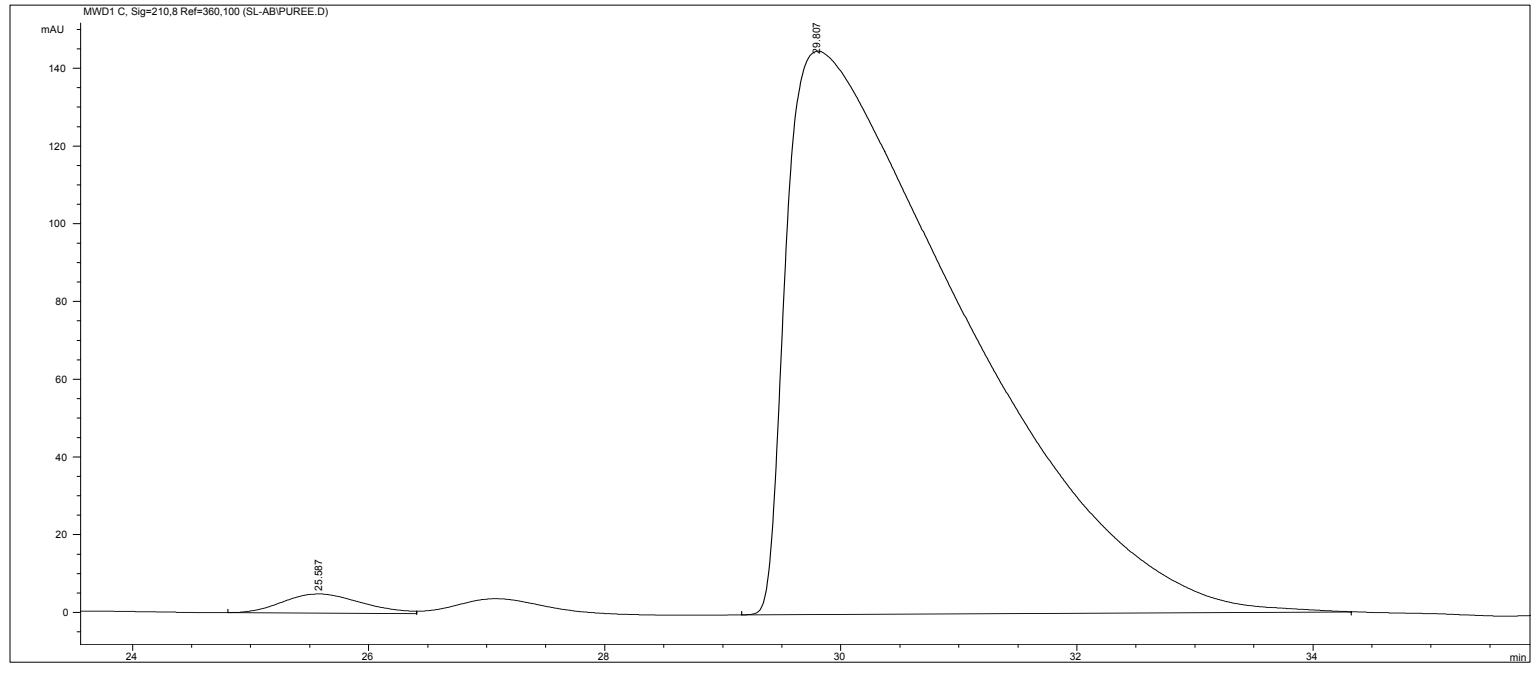

Signa1 3: MWD1 C, Sig=210,8 Ref $=360,100$
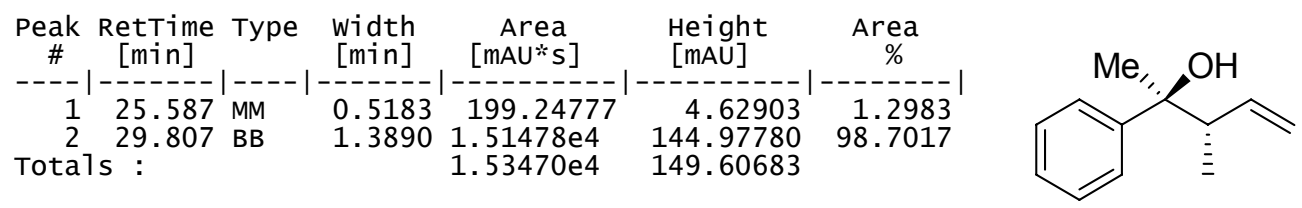
syn-13a: Chiralpak ${ }^{\circledR}$ AD-H Column, Hexane:IPA = 999:1, $1.0 \mathrm{~mL} / \mathrm{min}, 210 \mathrm{~nm}$

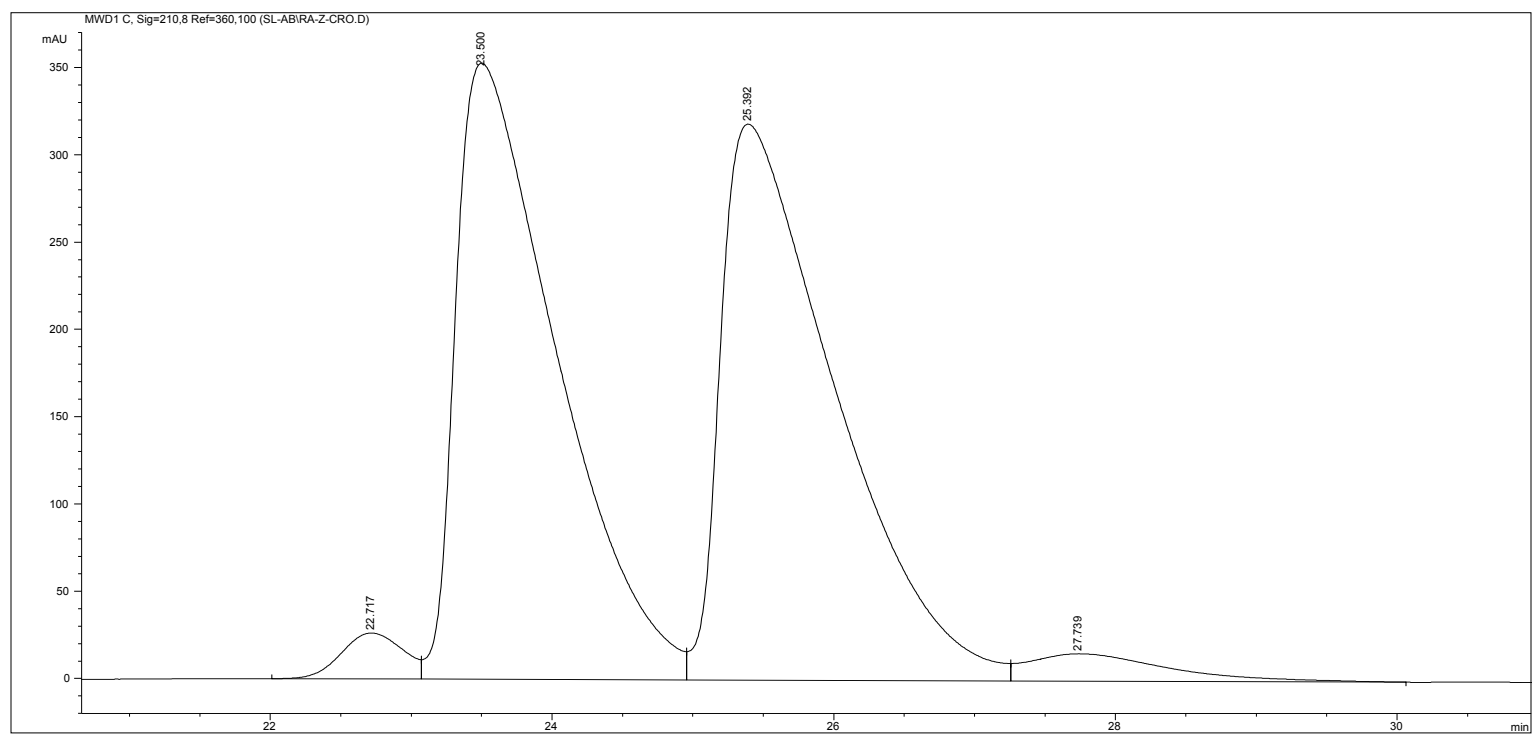

Signal 3: MWD1 C, Sig $=210,8$ Ref $=360,100$

\begin{tabular}{|c|c|c|c|c|c|c|}
\hline$\underset{\#}{\text { Peak }}$ & $\begin{array}{c}\text { RetTime } \\
{[\text { min] }}\end{array}$ & Type & $\begin{array}{l}\text { Width } \\
\text { [min] }\end{array}$ & $\begin{array}{c}\text { Area } \\
{[\mathrm{mAU} * \mathrm{~s}]}\end{array}$ & $\begin{array}{l}\text { Height } \\
\text { [mAU] }\end{array}$ & $\begin{array}{c}\text { Area } \\
\%\end{array}$ \\
\hline $\begin{array}{r}1 \\
2 \\
3 \\
4 \\
\text { Tota }\end{array}$ & $\begin{array}{l}22.717 \\
23.500 \\
25.392 \\
27.739 \\
5::\end{array}$ & $\begin{array}{l}\text { BV } \\
\text { VV } \\
\text { VV } \\
\text { VP }\end{array}$ & $\begin{array}{l}0.4735 \\
0.7358 \\
0.8265 \\
0.8588\end{array}$ & $\begin{array}{l}798.91400 \\
1.76076 \mathrm{e} 4 \\
1.80332 \mathrm{e} 4 \\
1070.03833 \\
3.75098 \mathrm{e} 4\end{array}$ & $\begin{array}{r}26.36596 \\
353.07904 \\
318.51807 \\
15.66738 \\
713.63045\end{array}$ & $\begin{array}{r}2.1299 \\
46.9415 \\
48.0760 \\
2.8527\end{array}$ \\
\hline
\end{tabular}
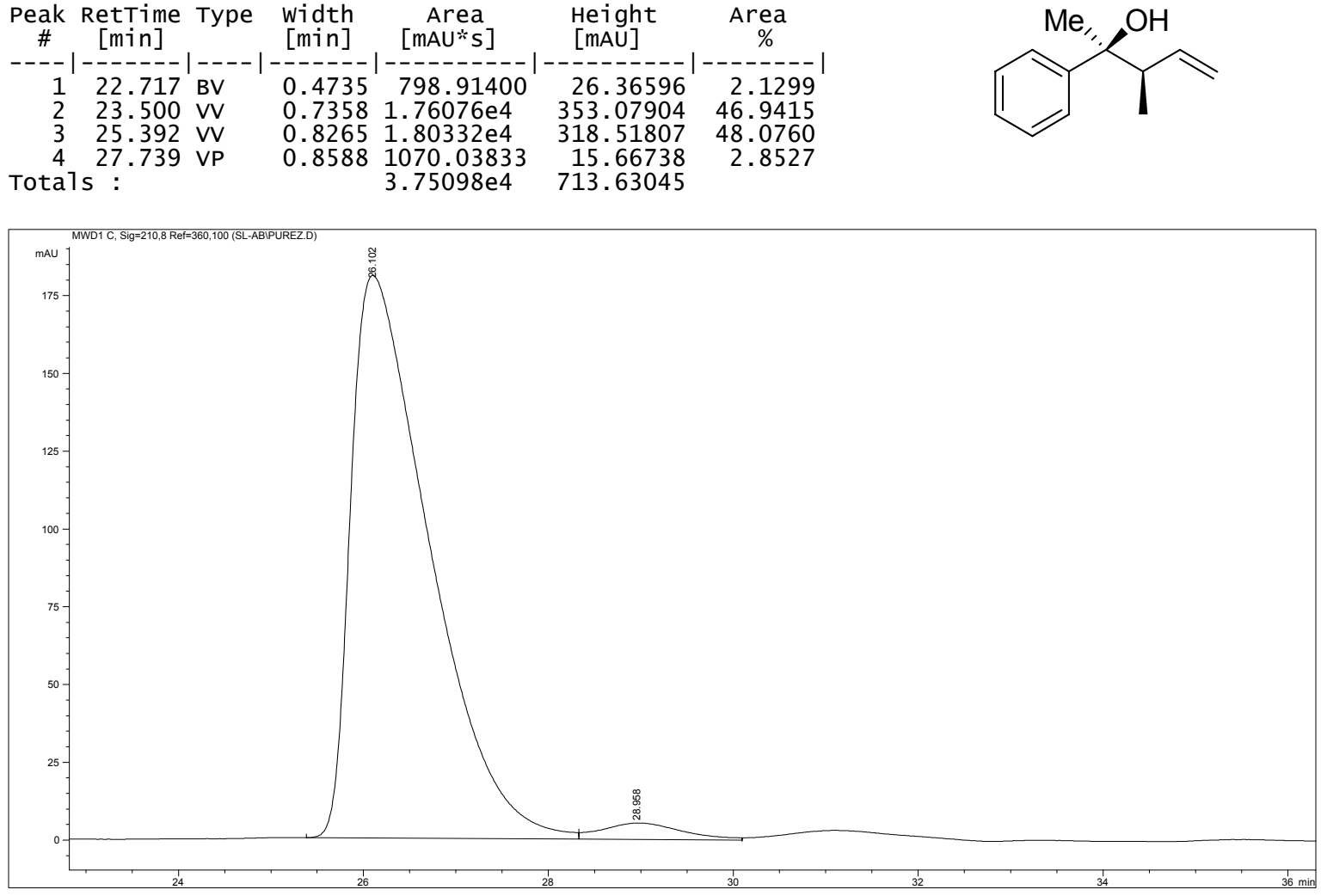

Signal 3: MWD1 C, Sig=210, 8 Ref $=360,100$
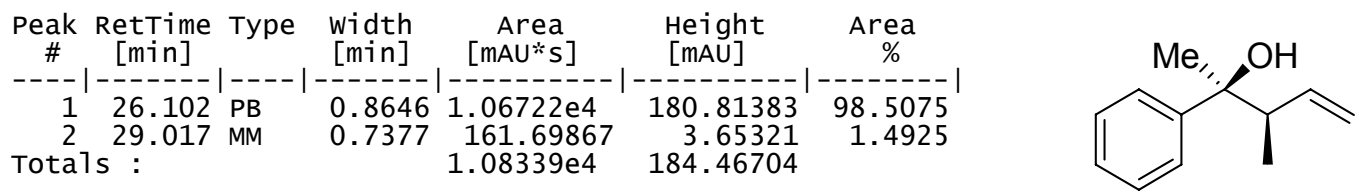